\title{
RELATIVISTIC SUPERFLUID MODELS FOR ROTATING NEUTRON STARS
}

\author{
Brandon Carter \\ Observatoire de Paris, 92195 Meudon, France. \\ Email: Brandon.Carter@obspm.fr \\ Chapter contributed to \\ PHYSICS OF NEUTRON STAR INTERIORS, \\ (Trento, June 2000), \\ ed. D. Blasche, N.K. Glendenning, A. Sedrakian
}

\begin{abstract}
This article starts by providing an introductory overview of the theoretical mechanics of rotating neutron stars as developped to account for the frequency variations, and particularly the discontinuous glitches, observed in pulsars. The theory suggests, and the observations seem to confirm, that an essential role is played by the interaction between the solid crust and inner layers whose superfluid nature allows them to rotate independently. However many significant details remain to be clarified, even in much studied cases such as the Crab and Vela. The second part of this article is more technical, concentrating on just one of the many physical aspects that needs further development, namely the provision of a satisfactorily relativistic (local but not microscopic) treatment of the effects of the neutron superfluidity that is involved.
\end{abstract}




\section{Elementary global mechanics of rotating neutron stars}

\subsection{Introduction.}

Long before their observational detection as pulsars, theoreticiens were well aware [1] of the special physical interest of neutron stars - whose existence was confidently predicted - as well as of the (still entirely speculative) possibility of other more exotic (e.g. strange) stars of comparable compactness, meaning a radius only a few times larger than the Schwarzschild limit value, $R=2 \mathrm{G} M / c^{2}$, for a mass comparable with that of our Sun. Having presumably been formed by collapse of a stellar core that marginally exceeds the Chandrasekhar limit for for a self gravitating body with insufficient thermal pressure, a typical neutron star can be expected to a have a mass rather close to this limit, which - in terms of Newton's constant $\mathrm{G}$, the speed of light $c$, the Dirac Planck constant $\hbar$, and the proton mass $m_{\mathrm{p}}$ - is given very roughly by the simple formula

$$
M \approx\left(\frac{\hbar c}{\mathrm{G}}\right)^{3 / 2} m_{\mathrm{p}}^{-2},
$$

whose derivation is based just on the supposition that $m_{\mathrm{p}}$ gives a rough estimate of the mass per cubic Fermi length, regardless whether the degenerate relativistic fermions in question are electrons (as in an ordinary white dwarf) neutrons, or even quarks.

Unlike what was possible when superfluidity of the neutron matter in such compact stars was originally predicted [2] by Migdal, present day article accelerators can explore the physics of individual particle at energies that are now approaching the order of a TeV. Nevertheless, although their levels - from $\mathrm{MeV}$ to at most the order of $\mathrm{GeV}$ - are only moderate by such modern standards, the thermal energies - and particularly the Fermi energies - characteristic of matter in neutron stars remain beyond the range accessible in the laboratory for bulk matter.

For a mass near the value given by (1), the condition that the stellar radius be large compared with the Schwarzschild value, $R=2 \mathrm{G} M / c^{2}$, places an upper bound

$$
\rho_{*} \ll\left(\frac{c}{\hbar}\right)^{3} m_{\mathrm{p}}^{4}
$$

on the mean stellar density $\rho_{*}$ - and hence also on the central density (since unlike what is possible other kinds of stars, a neutron star cannot have a density profile that is sharply peaked at the center). While less compact neutron star configurations (with lower mass and larger radius) can exist in principle, it is hard to see how they could be created in nature, so a typical example can be expected to have a central density that is not so very far below what is permitted by this Oppenheimer - Volkoff bound (2). Since this bound is interpretable as the order of a proton mass per cubic proton Compton length, it is evidently quite a lot higher than the density of the order of a proton mass per cubic pion Compton length that characterises ordinary nuclear matter. In terms of the pion mass $m_{\pi}$ this ordinary nuclear density will be given in order of magnitude by

$$
\rho_{\mathrm{nuc}} \approx\left(\frac{c}{\hbar}\right)^{3} m_{\pi}^{3} m_{\mathrm{p}},
$$

which is a few times $10^{14} \mathrm{gm} / \mathrm{cm}^{3}$. The prediction that typical neutron star core densities are thus well beyond what is easily accessible to experiment is one of the reasons why it is so interesting, not just for astronomy, but also for the basic physics [3, 4] of bulk matter at 
the corresponding intermediate energy levels, to acquire and analyse as much observational information as possible about neutron stars (as well as "strange" or other comparably compact stars, which, if they exist, will also have core densities in the same range).

In addition to the limited amount of such information that is available from other mechanisms (such as binary orbital behaviour), we are fortunate to have at our disposal an enormous and steadily increasing body of relevant information provided ( see Figure 1) by pulsar timing measurements: radio (and in some cases optical or other) observations provide continuous high (sometimes within $10^{-9}$ ) precision monitoring of pulsar frequencies, which are generally believed to correspond directly to the rotation frequency $\Omega$ of the underlying star, or more precisely to that of its rigidly rotating outer "crust".

The present article (like a briefer preceeding review [5]) is intended as a self contained introduction to the theory of the phenomena most relevant to such observations. It is meant to be accessible to non-specialist readers, who are assumed just to have a grounding in general physics, at the level provided by Landau and Lifshitz [6], in areas including relativistic gravitation theory, and non relativistic superfluidity and superconductivity theory.

As discussed in detail in accompanying articles in this volume, outside a still mysterious core (that may consist of quark matter) neutron stars are generally believed to consist essentially of a neutron fluid interior and a surrounding crust. The outer crust material is qualitatively similar to an ordinary metal, consisting of baryons concentrated (as a majority of neutrons with a minority of protons) in nuclei in a degenerate Fermi type sea of electrons at concentrations up to and beyond the white dwarf limit, where the electrons become relativistic, at a density given in terms of the electron mass $m_{\mathrm{e}}$ by

$$
\rho_{\mathrm{rel}} \approx\left(\frac{c}{\hbar}\right)^{3} m_{\mathrm{e}}^{3} m_{\mathrm{p}}
$$

whose value, of the order of $10^{7} \mathrm{gm} / \mathrm{cm}^{3}$, corresponds to about one proton mass per cubic electron Compton length.

The transition to the qualitatively different kind of material that makes the behaviour of neutron stars so very different from that of ordinary degenerate electron supported white dwarf stars occurs at a critical "neutron drip" density $\rho_{\text {drip }}$ that is reached when the Fermi energy of the degenerate electrons becomes comparable the binding energy $E_{\text {nuc }}$ per baryon in a nucleus, whose value is of the order of the Fermi energy of the protons and neutrons when their mean separation is of the order of a pion Compton length, i.e. $E_{\text {nuc }} \approx\left(m_{\pi} c\right)^{2} / 2 m_{\mathrm{p}}$. Above this density,

$$
\rho_{\text {drip }} \approx\left(\frac{c}{\hbar}\right)^{3}\left(\frac{m_{\pi}^{3}}{2 m_{\mathrm{p}}}\right)^{2},
$$

which works out to be a few times $10^{11} \mathrm{gm} / \mathrm{cm}^{3}$, the crust matter will still contain positively charged baryonic nuclei in a negatively charged Fermi sea of electrons, but there will now also be a third constituent consisting of freely moving neutrons outside the nuclei.

The use of the term "crust" to describe the layers both above and below the critical value (5) is motivated by the consideration that the ionic nuclei will crystallise as a Coulomb lattice whose large scale behaviour will be that of an elastic solid as soon as the star has cooled sufficiently. Except for a very thin outer surface layer with density below the white dwarf limit (4) that may remain liquid as a relatively shallow "ocean", the rest of the crust is expected [7] to have solidified by the time the neutron star has dropped below the MeV level, which will be reached within a few months of its formation. Due to the high conductivity of 
the degenerate electrons the outer magnetic field will be firmly anchored in this crust, whose rotation rate is therefore what is is measured directly by pulsar frequency observations.

For the purpose of explaining these observations (see Figure 1) the most interesting feature of the crust is the presence of the interprenetrating neutron fluid in the inner crust, at densities ranging from the critical "drip" value (5) all the way up to the nuclear value (3) that is reached at the base of the crust beyond which the ions dissolve. In the relatively low temperatures (below an $\mathrm{MeV}$ ) that are relevant it is generally believed [8, 9] that the unconfined neutrons (like those within the nuclei) will form Cooper type pairs that will form a bosonic condensate. The interpenetrating neutron constituent is thereby endowed with the property of superfluidity, which enables it to flow freely past the metallic lattice (and the electron sea to which the lattice is electrically coupled) in the manner illustrated schematically in the following diagram (using hyphens to indicate the negatively charged electrons, crossed circles to indicate the positively charged nuclei, and double arrows to indicate relatively moving Cooper pairs of neutrons):

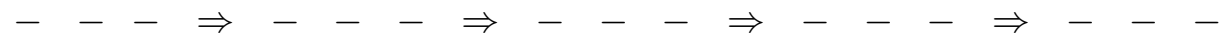

$$
\begin{aligned}
& -\Rightarrow---\bigoplus---\Rightarrow---\bigoplus---\Rightarrow- \\
& -\quad-\quad \Rightarrow-\quad-\quad \Rightarrow-\quad-\quad \Rightarrow \quad-\quad-\quad \Rightarrow-D_{-} \\
& -\Rightarrow---\Rightarrow-\quad-\quad \Rightarrow \quad-\quad-\quad \Rightarrow--\quad-\Rightarrow- \\
& -\quad-\Rightarrow-\quad-\quad-\Rightarrow-\quad-\quad \Rightarrow \quad-\quad-\quad-\Rightarrow-D_{-} \\
& -\bigoplus---\Rightarrow---\bigoplus---\Rightarrow---\bigoplus- \\
& -\quad-\Rightarrow---\Rightarrow---\Rightarrow---\Rightarrow-- \\
& -\Rightarrow---\Rightarrow---\Rightarrow-\quad-\Rightarrow---\Rightarrow- \\
& -\quad-\Rightarrow-\quad-\quad \Rightarrow--\quad-\Rightarrow-D_{-} \Rightarrow-{ }_{-} \\
& -\Rightarrow---\bigoplus---\Rightarrow--_{-}-\bigoplus---\Rightarrow-
\end{aligned}
$$

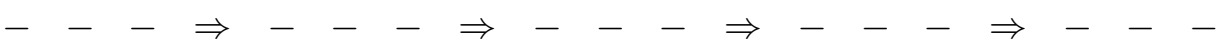

The unconfined neutrons thus constitute a massive component that can rotate independently of the crust, thereby - as explained below - providing the most promising kind of mechanism for explaining the observed pulsar frequency glitches (see Figure 1).

At the base of the inner crust, at densities above the nuclear value given by (3), it is generally believed that the neutron fluid and ionic constituents merge to form a uniform fluid composed mainly of (superfluid) neutrons but with an independently moving (superconducting) protonic constituent, and with the further complication [10] that instead of forming ordinary scalar Cooper type pairs the neutrons at this deeper level condense as pairs of spin 1. At even higher densities, beyond that of ordinary nuclear matter, various more or less exotic possibilities have been suggested, but no firm concensus has yet emerged. For example Glendenning has predicted [11, 12] that there will be a hybrid zone in which negatively charged drops of quark matter will condense within the surrounding positively charged baryonic liquid, and moreover that they will crystallise to form an ionic solid analogous to that of the crust. At even higher densities, as the maximum allowed by (2) is approached, one might expect that there would be an inner core where the drops merge to form another homogeneous superconducting superfluid zone, that (unlike the outer, baryonic core) would be constituted purely of quark matter, in which interesting new kinds of superfluidity and superconductivity [13, 14, 15, 16] could occur. Like the somewhat better understood inner crust and outer 


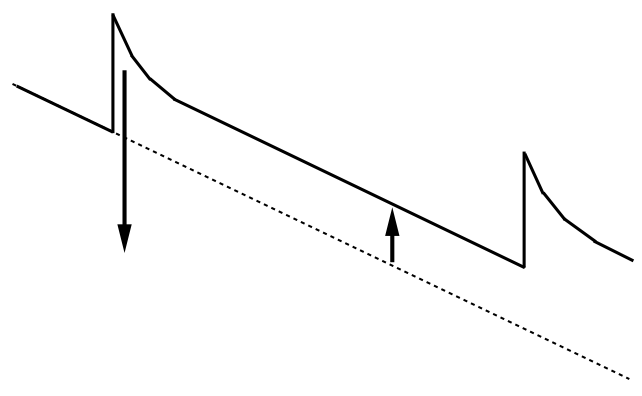

Figure 1: Qualitative sketch of a typical observational plot of pulsar angular velocity $\Omega$ against time $t$. The long down - pointing arrow indicates the negative change $\Delta \Omega$ during a period of steady slow down. The short up - pointing arrow indicates (on an exagerated scale) the positive jump $\delta \Omega$ during a glitch (consisting of a sharp discontinuity followed by a transient readjustment).

core regions, these very high density inner regions may also be relevant to the interpretation of pulsar frequency observations such as are illustrated in Figure 11.

The overall situation is not just that the global structure and behaviour of a neutron star is rather complicated, but furthermore that (as a fortiori for strange stars if they exist) many important aspects remain so unclear that - except in the crust region (or for very low mass neutron stars) for which a reasonable degree of concensus already prevails - it is hardly worthwhile yet even to start the detailed numerical calculations that will be needed later on. Before a convincingly realistic neutron star model can be developed even as a rough approximation many underlying physical issues will need to be dealt with, of which the most basic are concerned with the qualitative nature of matter at the supernuclear densities attained in the cores of all neutron stars above or near the precise Chandrasekhar limit value, $M \simeq \sqrt{2} M_{\odot}$ (where $M_{\odot}$ is the solar mass) though not for very low mass neutron star configurations (which are at least of academic interest, even though it is hard to see how they could be created in nature.

From the point of view of the interpretation of observational data, many less fundamental but technically non-trivial issues need to be be clarified. Among the other accessory issues (concerning matter at less extreme densities) that also need to be dealt with, the one with which the present article will be primarily concerned is that of the consequences of the predicted superfluidity. The final sections will concentrate on the results of recent progress on the development of an appropriately relativistic treatment as an improvement (in view of immediate coherence, as well as the long term objecive of precision) over the non-relativistic treatment that has until now been mainly used, not just for superfluidity but also for many other relevant phenomena. However before going into the technical aspects of the relativistic treatment, the first part of this article will provide a brief survey of the reasons why the phenomenon of superfluidity is particularly important for relating theoretical understanding of the inner structure of the neutron star to the available observational data, of which the most richly informative part (see Figure 1) comes from pulsar timing. 


\subsection{Minimal two component rotating star models}

As emphasised above, for many of the most important questions about the global structure of neutron stars, no quantitative agreement is available or to be expected for a long time yet. There are however several essential qualitative features on which practically all neutron star theorists do seem to agree already. In relation to the pulsar timing observations, the most important of these agreed features [7] is the presence of a rigidly corotating structure that may or may not include part of the core but certainly includes a solid outer crust to which is anchored the magnetic field configuration that gives rise to the observed radio and other emission. The next most important feature, common to all viable theoretical scenarios albeit of a more subtle nature, is the presence of some (maybe many) effectively superfluid (or superconducting) zones that can rotate independently [8] of the rigidly rotating crust structure whose angular velocity, $\Omega_{\mathrm{c}}$ say, is presumed to be the same as the $\Omega$ that is directly observed.

The mimimal agreement about these two essential features is what underlies the longstanding, widespread, and enduring popularity of a corresponding kind minimally complicated rotating neutron star model, involving just two independently rotating parts: a "corotating crust" part with (directly observed) angular velocity $\Omega_{\mathrm{c}}$ and a "superfluid neutron" part with a possibly different angular velocity $\Omega_{\mathrm{n}}$ (representing the average of what in a more detailed treatment would be a spacially variable angular velocity distribution).

The basic postulate of such a minimal model is that the total angular momentum $J$ of the star is the sum of decoupled parts,

$$
J=J_{\mathrm{c}}+J_{\mathrm{n}}
$$

with

$$
J_{\mathrm{c}}=I_{\mathrm{c}} \Omega_{\mathrm{c}}, \quad J_{\mathrm{n}}=I_{\mathrm{n}} \Omega_{\mathrm{c}},
$$

where $I_{\mathrm{c}}$ and $I_{\mathrm{n}}$ are separate moments of inertia that are supposed to remain constant during a process of continued variation governed by an external torque $\Gamma_{\text {ex }}$. (A more sophisticated variant of this model would allow for cross coupling, whereby $J_{\mathrm{c}}$ is affected by $\Omega_{\mathrm{n}}$ and vice versa: a small cross coupling of this kind would inevitably be present [17] in an exactly relativistic description, and a possibly more important cross coupling effect is to be expected from the effect - to be discussed below - of superfluid momentum "entrainment", whose likely relevance in neutron star matter was originally pointed out in the context of proton superconductvity [18, 19].)

The external couple $\Gamma_{\text {ex }}$ represents the effect of the magnetic field anchored in the rotating crust, which, in view of the high conductivity of the crust is generally assumed to remain constant over timescales long compared with those (at most a few years, since the oldest pulsar observations go back only to 1968) of the observed fluctations. The effect of this steady couple is of course to cause a total angular momentum loss rate given by

$$
\dot{J}=\Gamma_{\mathrm{ex}}<0 .
$$

If the angular velocities were locked together, $\Omega_{\mathrm{n}}=\Omega_{\mathrm{c}}=\Omega$, this would give $\dot{\Omega}=\Gamma_{\text {ex }} / I$ with

$$
I=I_{\mathrm{c}}+I_{\mathrm{n}},
$$

and this relation will in any case be true for the long term average, $\langle\Omega\rangle$ i.e. since (for reasons to be discussed below) the separate angular velocities can never get too far appart, the long 
term slowdown of the observed pulsar frequency allows the torque involved to be estimated as

$$
\Gamma_{\mathrm{ex}}=I\langle\dot{\Omega}\rangle \text {. }
$$

in which, for neutron stars with mass $M \simeq 3 M_{\odot} / 2$ (which, consistently with the theoretical prediction (1), is what has been found [20] for the few cases in which the mass is reliably measurable), the total moment of inertia (unlike the distinct parts $I_{\mathrm{n}}$ and $I_{\mathrm{c}}$ for which different theoreticians have rather diverse ideas in various cases) can be evaluated in a generally agreed manner, which leads [21] to estimates of about $10^{2} M_{\odot} \mathrm{Km}^{2}$ within a factor of order unity (whereas the uncertainty range would be much larger for a neutron star nearer the upper mass limit).

The idea of the two component model is that as well as supporting the effect of the external torque $\Gamma_{\text {ex }}$, the crust component exerts an internal torque $\Gamma_{\text {in }}$ on the "superfluid neutron" component, which therefore obeys an evolution equation of the form

$$
\dot{J}_{\mathrm{n}}=\Gamma_{\text {in }},
$$

while, in order to be consistent with (9) the crust component must obey

$$
\dot{J}_{\mathrm{c}}=\Gamma_{\mathrm{ex}}-\Gamma_{\mathrm{in}},
$$

in which, unlike the external torque $\Gamma_{\mathrm{ex}}$, the internal torque is not constant but proportional to the angular velocity difference

$$
\Gamma_{\text {in }}=-\frac{I_{\mathrm{n}} I_{\mathrm{c}}}{I} \frac{\omega}{\tau}, \quad \omega=\Omega_{\mathrm{n}}-\Omega_{\mathrm{c}},
$$

where $\tau$ is a damping timescale whose estimation will be discussed below. The chosen normalisation of this timescale is such that, according to the preceeding equations, the angular velocity difference will satisfy a differential equation of the simple form

$$
\dot{\omega}+\frac{\omega}{\tau}=-\frac{I}{I_{\mathrm{c}}}\langle\dot{\Omega}\rangle
$$

in which the right hand side is a constant that fixes the saturation limit

$$
\omega \rightarrow \omega_{\mathrm{s}}, \quad \omega_{\mathrm{s}}=-\frac{I}{I_{\mathrm{c}}}\langle\dot{\Omega}\rangle \tau>0,
$$

to which the difference $\omega$ will tend in the long run, unless this continuous evolution process is interrupted by a "glitch". So long as no such interruption has occurred, the angular velocity difference will be given as a function of the time $t$ by an expression of the form

$$
\omega=\omega_{\mathrm{s}}+\left(\omega_{0}-\omega_{\mathrm{s}}\right) \mathrm{e}^{-t / \tau},
$$

where $\omega_{0}$ is a constant of integration interpretable as the value of $\omega$ when the clock time $t$ was set to zero. 


\subsection{The problem of accounting for glitches}

Some of the strongest observational evidence in favour of the theoretical picture epitomised by the kind of highly simplified 2 component neutron star model described in the previous section is provided by a phenomenon in which the continuous evolution described by this model is subject to a temporary model break down. The phenomenon in question is what is known as a glitch (see Figure $\mathbb{1}$ ) during which (with a rise time too short to be measured, at most a few hours and probably much less) the observed angular frequency $\Omega$ of an isolated pulsar undergoes a positive discontinuity, $\delta \Omega>0$, that partially cancels the loss $\Delta \Omega$ during the preceeding period of continuous slowdown.

Since there is no imaginable way the external torque could suddenly become very large (nor any observational evidence that the associated pulsar emission process changes significantly at all during a glitch) there can be no corresponding discontinuity in the angular momentum. This means that, if we want to use a model only involving a single component, it will be necessary to take account of variation of the moment of inertia, whose total $I$ will evidently undergo a negative variation $\delta I$ given, for a glitch of amplitude $\delta \Omega$, by

$$
\delta J=0 \Rightarrow \delta I=-I \frac{\delta \Omega}{\Omega} .
$$

The earliest theory designed to account for this phenomenon, as first observed in the Crab and Vela pulsars, was based on the first of what were presented in the preceeding section as generally agreed features of neutron stars, namely the presence of a solid crust structure, but on the basis of a single component model taking no account of the second generally agreed feature (namely the possibility of independently rotating parts). The idea [22, 8, 23] was that the rigidity of the solid crust would tend to prevent the decrease in moment of inertia that would necessarily accompany the loss of angular momentum in a purely fluid star. In a simple rotating fluid star model, the oblateness due to centrifugal force would give rise to a variable moment of inertia that would be expressible for low values of the angular velocity by an expression of the quadratic form

$$
I \simeq I_{0}\left(1+\frac{\Omega^{2}}{\Omega_{*}^{2}}\right)
$$

where $I_{0}$ is the value of the moment of inertia in the non-rotating spherical limit and $\Omega_{*}$ is a constant specifying the relatively high value of the angular velocity (which will be given in terms of the mean density subject to (2) by the rough order of magnitude estimate $\Omega_{*}^{2} \approx$ $\mathrm{G} \rho_{*}$ ) that would be necessary for deviations from spherical symmetry to be of order unity. Whatever may have happened immediately after the birth of the neutron star, no such rapid rotation still occurs in any of the (at least centuries old) pulsars that are actually observed today, for which the condition $\Omega^{2} \ll \Omega_{*}^{2}$ is always satisfied.

For a simple perfect fluid star model, the effect of the external torque (8) during an extended time interval $\Delta t$ would be to cause an angular momentum loss, $\Delta J \simeq \Gamma_{\mathrm{ex}} \Delta t<0$ that would be accompanied by a corresponding angular velocity variation $\Delta \Omega<0$, which according to (18) would entail a decrease in moment of inertia given by

$$
\Delta I \simeq 2 I \frac{\Omega^{2}}{\Omega_{*}^{2}} \frac{\Delta \Omega}{\Omega}<0 .
$$


Due to the solidity of the crust, which tends to preserve the more highly elliptic initial configuration, the actual change in the moment of inertia will fall short of what is predicted by this formula, but at some stage the strain will build up to the point at which the solid structure will break down. The implication is that there will then be a "crustquake", in which the solid structure suddenly changes towards what the perfect fluid structure would have been, thereby changing the moment of inertia by an amount that will be at most of the order of the upper limit given by

$$
\delta I \lesssim \Delta I,
$$

where $\Delta I$ is what is given by (19), and that will be considerably less than this if the crust rigidity is low. According to (17) the corresponding positive angular velocity discontinuity $\delta \Omega$ associated with the continuous negative angular velocity change $\Delta \Omega$ since the preceeding glitch will be subject to the limit

$$
\delta \Omega \lesssim-2 \frac{\Omega^{2}}{\Omega_{*}^{2}} \Delta \Omega
$$

The preceeding formula provides an order of magnitude limit that must be satisfied by a rather large margin if the rigidity is low but that is entirely consistent with what is observed in the case of the Crab pulsar, for which typical glitches are characterised by $\delta \Omega \approx 10^{-8} \Omega$. However almost immediately after it was first proposed, it began to be recognised [7] that this rather obvious mechanism would not be sufficient to account for the much larger glitches that are frequently observed in cases such as that of the Vela pulsar, for which typical glitch amplitudes are characterised by $\delta \Omega \approx 10^{-6} \Omega \approx-10^{-2} \Delta \Omega$.

Since it was first suggested by pioneers such as Anderson and Itoh [24, the generally accepted way of getting round this limitation - namely that the likely changes of the moments of inertia will be far too small to account for frequent giant (Vela type) glitches - is to drop the single component description in favor of the two component description in which glitches can be accounted for even if (as assumed in its simplest version) the relevant moments of inertia undergo no significant change at all. The essential point is that the consideration that the very short glitch duration excludes any significant jump in the total angular momentum does not rule out the possibility of impulsive transfer of angular momentum between the two components provided they balance out:

$$
\delta J=0 \quad \Rightarrow \quad \delta J_{\mathrm{c}}=-\delta J_{\mathrm{n}}
$$

so that

$$
\delta \Omega_{\mathrm{n}}=-\frac{I_{\mathrm{c}}}{I_{\mathrm{n}}} \delta \Omega_{\mathrm{c}}
$$

The idea is that between the glitches the weak coupling mechanism described by (14) allows the slowdown of the "neutron superfluid" angular velocity $\Omega_{\mathrm{n}}$ to lag behind that of the crust component which is what is presumed to be actually observed, $\Omega=\Omega_{\mathrm{c}}$, so that during the preceeding period $\Delta t$ the angular velocity difference $\omega$ will be positive. It is generally supposed that (for diverse reasons to be discussed below) this angular velocity difference gives rise to stresses that are partially relaxed in the glitch process, whose onset occurs when the difference $\omega$ reaches a critical glitch inducing value $\omega_{\mathrm{g}}$ say that, in order to be attainable must be less than the limit $\omega_{\mathrm{s}}$ given by (15) - a condition that would fail if the relaxation timescale $\tau$ were too short. 
Leaving aside cases for which $\omega_{\mathrm{g}}>\omega_{\mathrm{s}}$ (whose evolution will be of the glitch free kind recently investigated [25] by Sedakian and Cordes) as well as the marginal case in which $\omega_{\mathrm{g}} \approx \omega_{\mathrm{s}}$, i.e. subject to the proviso that there is a safe margin $\omega_{\mathrm{g}} \ll \omega_{\mathrm{s}}$, the evolution equation (16) will be replacable by the linear relation

$$
\frac{\omega}{\omega_{\mathrm{s}}}=\frac{\omega_{0}}{\omega_{\mathrm{s}}}+\frac{t}{\tau}
$$

in which each of the terms is small compared with unity. Successive glitches bring about negative adjustments $\delta \omega$ that are needed to cancel out the cumulative effect of the positive variations $\Delta$ that develop during the duration of the interglitch periods governed by (24), so that on average they cannot deviate too much from the order of magnitude estimate given simply by

$$
\delta \omega \approx-\Delta \omega,
$$

in which, by (15) and (24), the deviation built up during an interglitch interval of duration $\Delta t$ will be given simply by

$$
\Delta \omega=-\frac{I}{I_{\mathrm{c}}}\langle\dot{\Omega}\rangle \Delta t
$$

Using (23) to eliminate the unobservable jump $\delta \Omega_{\mathrm{n}}$ from the difference $\delta \omega=\delta \Omega_{\mathrm{n}}-\delta \Omega_{\mathrm{c}}$, the magnitude of the observable jump $\delta \Omega_{\mathrm{c}}$ can be estimated by (25) as

$$
\delta \Omega_{\mathrm{c}} \approx \frac{I_{\mathrm{n}}}{I} \Delta \omega .
$$

Since the observable interglitch frequency variation will be given roughly by $\Delta \Omega_{\mathrm{c}} \simeq\langle\dot{\Omega}\rangle \Delta t$ one sees from (26) and (27) that it provides a corresponding estimate

$$
\delta \Omega_{\mathrm{c}} \approx-\frac{I_{\mathrm{n}}}{I_{\mathrm{c}}} \Delta \Omega_{\mathrm{c}}
$$

for the observable frequency jump during a glitch. The presumption that $\Omega_{\mathrm{c}}$ is identifiable with the $\Omega$ that is observed allows us to compare this with the previous upper limit (21) that was obtained for the single component model with variable moment of inertia. It can be seen that the difference is simply that the small factor $\left(\Omega / \Omega_{*}\right)^{2}$ in the upper limit for the single component model is replaced, in the two component fixed moment of inertia model, by the ratio $I_{\mathrm{n}} / I_{\mathrm{c}}$ whose value is highly uncertain (in view of our lack of firm knowledge about what goes on in the core of the neutron star) but can plausibly be supposed to be of the order of unity, which is what is needed to account for the frequent very large glitches (with $\left.\delta \Omega \approx-10^{-2} \Delta \Omega\right)$ that are observed in examples such as Vela.

\subsection{The question of pinning and the damping timescale}

The foregoing estimate (28) is not sensitive to the particular value of the damping timescale $\tau$ except that it is assumed to be large compared with the interglitch period $\Delta t$ which is usually several months or more. This requirement might at first sight appear to be incompatible with observations of post glitch relaxation, in which shorter timescales of only a few weeks have been shown to be involved. Such discrepancies are however to be expected on the basis of

our general qualitative understanding [26] of what is involved. The strong density gradients 
in the star imply the existence of many different zones in which differential rotation with a wide range of damping timescales can occur. Our simplified two component description of glitches depends on taking the part with moment of inertia $I_{\mathrm{n}}$ to correspond to a substructure for which the relevant damping timescale timescale $\tau$ is very large. A formally similar two constituent model might also be used for describing post glitch relaxation with much shorter timescales, but for such an application the substructure with moment of inertia $I_{\mathrm{n}}$ would have to be reinterpreted as corresponding to some other part of the star. Of course if we wanted to describe both the glitches and the postglitch relaxation, in a single coherent framework, we would need to amalgamate the separate two component models so as to obtain a more elaborate model (such as has recently been used 27] for the analysis of precession) with three or more independent components (and with not just a single damping timescale but an antisymmetric matrix of mutual damping coefficients). Although the construction of such composite models is straightforward in principle, most authors have so far (quite reasonably) preferred to concentrate on particular aspects for which a less intricate description is adequate.

Even for applications, such as the glitch model of the previous section, for which a two component description is adequate as a lowest order approximation, the estimation of the relevant damping timescale remains a subject of great uncertainty. The situation has however been clearer since the general question of quasi stationary equilibrium in a rotating superfluid was systematically addressed in the context of neutron stars by Alpar and Sauls [28], Bildsten and Epstein [29] and the Sedrakians [30], who drew attention to the consideration that long damping timescales can arise not just from weak but also from strong coupling. These authors considered the basic general problem of a two constituent superfluid model of the simplest kind in a local configuration of differential rotation about a fixed axis characterised by a unit 3 vector, $\vec{\nu}$ say in the neighbourhood of a position determined (in a Newtonian flat space description) with respect to a central rotation axis by an orthogonal radius vector $\vec{r}$. One of the constituents is the "normal" (and therefore in a $n$ equilibrium state) rigidly rotating) crust constituent characterised by a (uniform) angular velocity $\Omega_{\mathrm{c}}$ and a corresponding velocity vector given as the cross product $\vec{v}_{\mathrm{c}}=\Omega_{\mathrm{c}} \vec{\nu} \times \vec{r}$. The large scale averaged velocity of the superfluid constituent - which for our purpose is to be thought of as constituted of neutrons is characterised in terms of a perhaps radially variable angular velocity $\Omega_{\mathrm{n}}$ by a similar formula $\vec{v}_{\mathrm{n}}=\Omega_{\mathrm{n}} \vec{\nu} \times \vec{r}$. However in the latter case it is to be born in mind that that on a microscopic scale the superfluid fluid is irrotational except on quantised vortex lines round which the integral of the relevant superfluid particle momentum $m v_{\mathrm{n}}$ is given by the Planck constant, i.e. $2 \pi \hbar$ in Dirac's notation, so that the corresponding velocity circulation is $\kappa=2 \pi \hbar / m-$ while, in view of the bosonic pairing, the relevant mass scale in the application with which we are concerned is twice that of the neutron, i.e. $m=2 m_{\mathrm{n}}$

Since the averaged velocity circulation per unit area orthogonal to the rotation direction $\vec{\nu}$ will simply be $2 \Omega_{\mathrm{n}}$, it follows that the corresponding surface number density, $\sigma$ say, of quantised vortices will be given by

$$
\sigma=\frac{\Omega m}{\pi \hbar}
$$

Although the superfluid motion is non dissipative, and so has no direct interaction with the "normal" crust material, the vortex cores (which are defects where the superflidity breaks down) will in general be subject to a drag force $\vec{F}_{\mathrm{d}}$ say per unit length, exerted by the bacground in the direction of relative motion. Using the notation $\vec{v}_{\mathrm{v}}$ for the velocity of motion of the vortex lines orthogonally to the rotationaxis $\vec{\nu}$ the dissipative drag force exerted by the 
background will be given by a formula of the form

$$
\vec{F}_{\mathrm{d}}=\eta_{\mathrm{r}}\left(\vec{v}_{\mathrm{c}}-\vec{v}_{\mathrm{v}}\right)
$$

in which $\eta_{\mathrm{r}}$ is a positive resistive drag coefficient, whose quantitative evaluation is a subject of much uncertainty - not just in the core, but even in the crust, where it is very sensitive to temperature [31] and other quantities such as superfluid pairing correlation lengths that are rather difficult to estimate [32].

This drag will not be the only force acting on the vortex line, which will also be subject to the Magnus effect. According to the well known formula [6] of Joukowski (or, in an alternative transliteration, Zhukovskii) this gives rise to a lift force $\vec{F}_{1}$ per unit length that is proportional to the product of the particle number density and the corresponding momentum circulation:

$$
\vec{F}_{1}=2 \pi \hbar n\left(\vec{v}_{\mathrm{n}}-\vec{v}_{\mathrm{v}}\right) \times \vec{\nu}
$$

where $n$ is the relevant particle number density. In the neutron superfluid application with which we are concerned the effect of the bosonic pairing must be taken into account, which means that the relevant number density is only half that of the neutrons themselves, i.e. $n=n_{\mathrm{n}} / 2$.

On the microscopically very long timescales characterising the relevant applications the vortex lines can be treated as effectively massless, which means that the evolution of the system will be determined simply by the condition that the total force on a vortex line must cancel out,

$$
\vec{F}_{\mathrm{d}}+\vec{F}_{\mathrm{l}}=0
$$

To solve this, it is convenient to decompose the velocity $\vec{v}_{\mathrm{v}}$ of the vortex lines into a (small) radially outward directed part, with magnitude $\dot{r}$, and a (larger, but for our purpose less important) remainder, directed parallel to the fluid flow vectors, in the form

$$
\vec{v}_{\mathrm{v}}=\frac{\dot{r}}{r} \vec{r}+\Omega_{\mathrm{v}} \vec{\nu} \times \vec{r}
$$

where $\Omega_{\mathrm{v}}$ is interpretable as the angular velocity of the vortex lattice. It is also convenient to introduce a dimensionless resistivity coefficient defined by

$$
c_{\mathrm{r}}=\frac{\eta_{\mathrm{r}}}{2 \pi \hbar n}
$$

which is what in the jargon of aero engineering would be called the drag to lift ratio (what, in that context, one seeks to minimise by cunning aerofoil design). The solution of (33) is thereby expressible as the condition that the vortex line angular velocity is intermediate between those of the crust and supefluid constituents, with value given by

$$
\Omega_{\mathrm{v}}=\frac{\Omega_{\mathrm{n}}+c_{\mathrm{r}}^{2} \Omega_{\mathrm{c}}}{1+c_{\mathrm{r}}^{2}}
$$

while the radially outward "creep" component of the velocity of the vortex lines will be given by

$$
\dot{r}=\frac{r c_{\mathrm{r}}}{1+c_{\mathrm{r}}^{2}}\left(\Omega_{\mathrm{n}}-\Omega_{\mathrm{c}}\right) .
$$


This last equation is particularly important because it determines the rate of change of the vortex line surface density $\sigma$ : as the comoving radius of the vortex distribution increases, the surface density will evidently undergo a corresponding decrease given by the relation $\dot{\sigma} / \sigma=-2 \dot{r} / r$. Since by (29) this surface density is proportional to the superfluid angular velocity, we deduce that the rate of variation of the latter will be given by

$$
\frac{\dot{\Omega}_{\mathrm{n}}}{\Omega_{\mathrm{n}}}=-\frac{2 c_{\mathrm{r}} \omega}{1+c_{\mathrm{r}}^{2}},
$$

where $\omega$ is the angular velocity difference as introduced in the relation (13), from which, by comparison with (37), the corresponding value of the damping timescale $\tau$ in which we are interested in, can be read out as

$$
\tau=\frac{I_{\mathrm{c}}}{2 I \Omega_{\mathrm{n}}}\left(c_{\mathrm{r}}+\frac{1}{c_{\mathrm{r}}}\right) .
$$

As well as showing that the timescale is subject to a lower limit (that might have been guessed on dimensional grounds) given by $\tau \geq I_{\mathrm{c}} / I \Omega_{\mathrm{n}}$ and attained for $c_{\mathrm{r}}=1$, a noteworthy feature of this result [28, 29, 30], is the dual symmetry between the roles of the drag to lift ratio $c_{\mathrm{r}}$ and of its inverse, the lift to drag ratio $c_{\mathrm{r}}^{-1}$. The decay timescale $\tau$ becomes infinitely large not just in the drag free limit for which $\eta 8 \mathrm{r}$ and hence $c_{\mathrm{r}}$ become arbitrarily small - so that by (35) the vortices are dragged allong with the superfluid - but also in the opposite "pinned" limit of very large $c$, for which the force on the vortices is strong enough to lock them to the crust material.

The estimation of the actual values of the $c_{\mathrm{r}}$, in the various zones of interest, has been the subject of much work, but the subject is difficult and many of the results are still inconclusive or controversial. Following the recognition [28] that magnetic coupling forces between the crust and the superconducting proton neutron superfluid zone are more important than had been previously supposed, and strong enough to lock this core region to the crust on timescales short compared with those relevant to glitch observations, it was suggested [33] that even the new coupling force values were underestimated, so much so that $c_{\mathrm{r}}$ would become large compared with unity, with the implication that $\tau$ could be very long after all, just as had been supposed in the early years when $c_{\mathrm{r}}$ had been supposed to be small. Even in the qualitatively more familiar crust regime the situation is still unclear, partly because of effects of temperature dependence: work of Jones [32, 34] suggests that pinning may be much less effective than had been previously supposed so that instead of being high $c_{\mathrm{r}}$ would be very low there.

\subsection{The long term crustal drift phenomenon}

The question of the effectiveness of vortex pinning to the crust comonent leads on to the related issue of what is actually responsible for the stress whose release, when a critical value is exceeded, is supposed to provide the glitch mechanism in the two constituent scenario described in the preceeding section. All the early versions of such a two constituent mechanism assumed that the relevant stresses would be due to vortex pinning. Like their more recent variants, the various early versions were classifiable in two distinct categories. In the first category [24, 35] it was supposed that the discontinuous breakdown would occur when some maximum static pinning force value was exceeded: the sudden (rather than "creeping") nature of the breakdown was accounted for, in a recent version [36], as being due to a thermal 
instability resulting from the temperature sensitivity of $c_{\mathrm{r}}$, while another new suggestion 37] is that the relevant slippage occurs at the locus where the ions dissolve at the base of the crust. In the second category [24, 38, 39] it was suggested that such a maximum pinning force value might never be reached because the elastic solid structure would breakdown first in a crustquake (of the kind required in the single constituent moment of inertia changing mechanism that may account for cases such as that of the Crab).

In all these various versions, the necessary transfer of angular momentum from the relevant independently rotating layers with moment of inertia $I_{\mathrm{n}}$ to the crust component with moment of inertia $I_{\mathrm{c}}$ is mainly attributable to the torque exerted by the pinning forces. However it has recently been pointed out [40] that there is an alternative possibility (effectively a new variant within the second category) whereby the necessary angular momentum transfer may be acheived convectively - by a transfer of matter (removal from the crust of matter with low angular momentum, and its replacement by matter with higher angular angular momentum) which can occur even if torque forces are entirely absent, i.e. in the small $c_{\mathrm{r}}$ limit. In this new kind of scenario, the stress ultimately responsible (when a critical level has been exceeded) for the discontinuous transfer is attributable to a centrifugal buoyancy deficit in the relatively slowed down crust component.

In the case of the earlier pinning driven mechanism, it was pointed out by Ruderman 39] that if the glitches were due to breakdown of the solid structure (rather than discontinuous vortex slippage) then the long term effect of many glitches would be analogous to that of terrestrial contentinental drift. It would give rise to a pattern of convective circulation 41] involving "transfusion" of matter from the crust constituent to the underlying neutron superfluid constituent in a "subduction" region near the equator, at colatitude $\theta=\pi / 2$, and the other way round near the poles at colatitude $\theta=0$. The corresponding long term average rate $\langle\dot{\theta}\rangle$ of angular drift of a crust plate at the surface, which for the spin down of an isolated pulsar would be directed away from the pole towards the equator (see Figure 2), was estimated by Ruderman on the assumption that it would correspond to an outward velocity of the same order of magnitude as the mean cylindrical expansion rate, $\langle\dot{r}\rangle \approx-\langle\dot{\sigma}\rangle / 2 \sigma$ of the vortex distribution, whose surface number density $\sigma$ is given in terms of the angular velocity by the proportionality relation (29). This reasonning [39] provided a formula of the form

$$
\langle\dot{\theta}\rangle \approx-\frac{\langle\dot{\Omega}\rangle}{2 \Omega}
$$

which implies that the timescale for complete turnover of the crust material is of the same order as the spin down lifetime of the pulsar, during which, as Ruderman pointed out, the magnetic dipole would be able to be dragged most of the way from the rotation axis to the equator. This would result in a net increase (ocurring discontinuously at the glitches) of the pulsar radiation rate and thus of the magnitude of the spin down rate $\dot{\Omega}$. Another reason for an increase of the spin down rate would be the decrease in oblateness according to (18), but this would evidently be much less important.

Unlike the original single constituent mechanism [22, 8, 23, 7] based on the loss of moment of inertia due to decrease in oblateness, and unlike the versions [35, 36, 37] of the twoconstituent theory that attribute the glitches to discontinuous vortex slippage, but like the Ruderman version [38, 39] (that applies when the pinning is too strong to be broken) the newly proposed two-constituent mechanism [40] (that applies when the pinning is too weak to be effective) will also entail a substantial rate of long term drift of plates of crust material. 


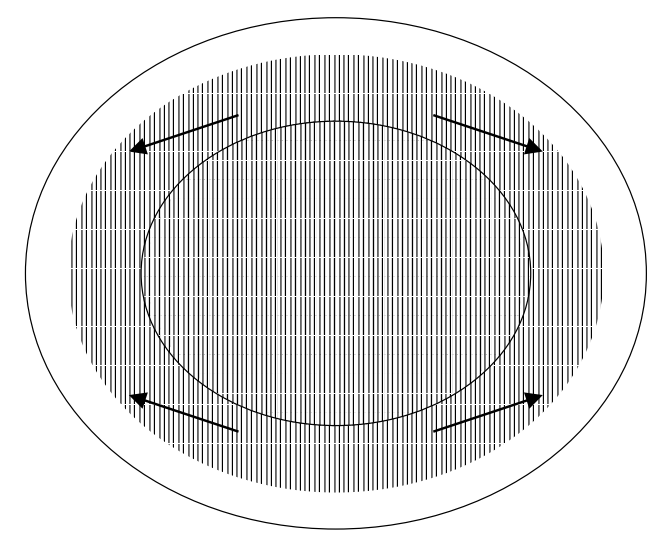

Figure 2: Qualitative sketch indicating direction of force expected (c.f Ruderman 1991) to act on (magnetically slowed down) crust due to vortex pinning mechanism, if it is effective, when the (interpenetrating) neutron superfluid retains a higher rotation rate. (Vertical shading indicates the alignement of the vortices in the region occupied by neutron superfluid, which is not confined to the core but interpenetrates the greater part of the solid cust as well.)

However this centrifugal buoyancy deficit mechanism differs from Ruderman's pinning driven mechanism in a manner that may be experimentally observable, since it is expected to produce plate drift in just the opposite direction, meaning that of decreasing colatitude $\theta$ for an isolated spinning down pulsar (see Figure 3), entailing transfusion of matter into the crust constituent near the equator, and out of it nearer the poles where $\theta$ is small. In this mechanism (unlike Ruderman's) the angular momentum of a crust plate will not be significantly changed when its colatitude undergoes a displacement $\delta \theta$ during a glitch, so its change of rotation frequency can be estimated as being given roughly by $\delta \Omega / 2 \Omega \approx-\delta \theta$ where $\delta \Omega$ is the glitch amplitude that is actually observed, and that partially cancels the preceeding interglitch variation $\Delta \Omega$. The change observed in the long run is the sum over the glitches of the combination $\Delta \Omega+\delta \Omega$, which will be the same as the sum of the hidden changes $\delta_{\mathrm{n}}$ (since the interglitch variation $\Delta_{\mathrm{n}}$ of the relevant superfluid part is assumed to be negligible). Since $\delta \Omega_{\mathrm{n}}=-\left(I_{\mathrm{c}} / I_{\mathrm{n}}\right) \delta \Omega$, by (23), it can be seen to follow that the long run average of the angular drift rate will be given by

$$
\langle\dot{\theta}\rangle \approx \frac{I_{\mathrm{n}}}{I_{\mathrm{c}}} \frac{\langle\dot{\Omega}\rangle}{2 \Omega}
$$

which has the opposite sign to what is given by the Ruderman formula (39), but can be comparable in magnitude since $I_{\mathrm{n}}$ can be comparable with $I_{\mathrm{c}}$. Indeed, in a case for which the moment of inertia $I_{\mathrm{n}}$ of the hidden part is large compared with the crust contribution, the magnitude given by the new formula (40) would be correspondingly larger than in the previous case, with the implication that the crust material would be entirely recycled several times during the spin down lifetime of the star, while this lifetime itself would presumably be considerably prolonged because the magnetic dipole axis would be dragged towards the pole, thereby decreasing the pulsar radiation rate.

For the purpose of observational discrimination between cases involving strong [38, 39], moderate [35, 36], or very weak [40], coupling the relevant directly measurable parameter is 


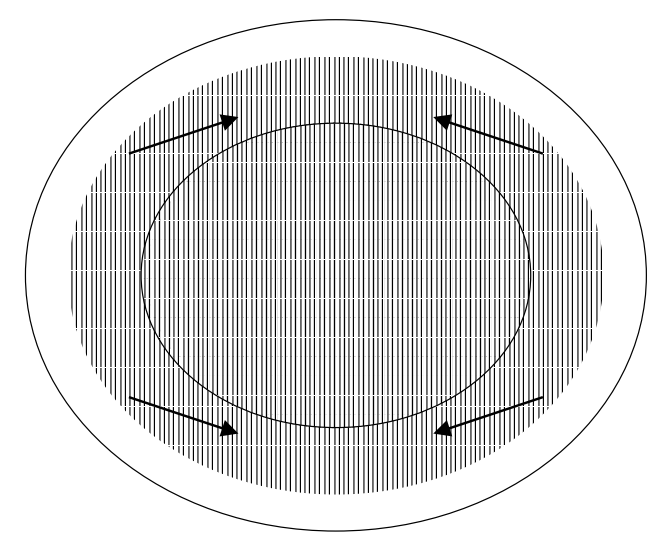

Figure 3: Qualitative sketch (using same shading conventions as before) indicating direction of force expected to act on (magnetically slowed down) crust, even if vortex pinning is ineffective, due to centrifugal buoyancy mechanism when the (interpenetrating) neutron superfluid retains a higher rotation rate.

what is known [42] as the (long term) braking index,

$$
\mathrm{n}=\frac{\langle\Omega\rangle\langle\ddot{\Omega}\rangle}{\langle\dot{\Omega}\rangle^{2}},
$$

and more particularly the braking deficit

$$
\epsilon=3-\mathrm{n}
$$

between the value that is observed and the value, $\mathrm{n}=3$, that is predicted [43] for a simple, rigid, non aligned magnetic dipole model, and also for more sophisticated pulsar emission models including allowance [44] for outflow of charged particles. If it is assumed that particle outflow and changes of moment of inertia can be neglected, then according to the simple dipole model [43] the relative spin down rate $\dot{\Omega} / \Omega$ is just proportional to $(\Omega \sin \alpha)^{2}$ where $\alpha$ is the dipole misalignment angle, i.e. the colatitude of the magnetic pole, so for this case the difference (42) can be immediately evaluated as

$$
\epsilon \simeq-\frac{2 \Omega\langle\dot{\alpha}\rangle}{\tan \alpha\langle\dot{\Omega}\rangle}
$$

When it gets near the extreme polar or equatorial values, $\alpha \simeq 0$, or $\alpha \simeq \pi / 2$, the evolution of the misalignment angle will of course have to come to a halt, $\langle\dot{\alpha}\rangle \simeq 0$, but in the intermediate range, i.e. for $\tan \alpha \approx 1$ one would expect the misalignment angle to move with the crustal drift, $\langle\dot{\alpha}\rangle \simeq\langle\dot{\theta}\rangle$. Subject to this assumption, the Ruderman formula (39) for the strong pinning model leads to the positive estimate

$$
\epsilon \approx \cot \alpha
$$

while the formula (40) for the model with negligible pinning gives the negative estimate

$$
\epsilon \approx-\frac{I_{\mathrm{n}}}{I_{\mathrm{c}}} \cot \alpha .
$$


The effects of variation of the moment of inertia [45] should not substantially effect the validity of these estimates except for new born pulsars with extremely rapid rotation, but electromagnetic effects of various kinds [46, 47] (including the obvious possibility of magnetic field decay) are more likely to give significant, typically positive, contributons to $\epsilon$. (In glitch free scenarios, Sedrakian and Cordes [25] have pointed out that differential rotation may bring $\epsilon$ down to negative values for periods of limited duration, but this sort of effect can be expected to cancel out in a long term average over many gliches).

If these estimates are indeed applicable, then in the case of the Vela pulsar the observed value [49], namely $\epsilon \simeq 3 / 2$, can be plausibly construed as evidence favouring the Ruderman model, with a moderate misalignement angle of the order of 40 degrees. A less clear cut case example is that of the Crab, for which the observed value [48], namely $\epsilon \simeq 1 / 2$, is also positive but considerably smaller, which suggests that this may be another instance to which Ruderman model applies, though with a relatively high misalignment angle. However in view of the above mentionned likelihood [46, 47] of other of other positive contributions to $\epsilon$, this evidence is too inconclusive to exclude the possibility that the Crab glitches may, after all, be attributable a slippage mechanism [35, 36, 37] of the first category, or even to the original simple oblateness mechanism subject to (21).

What transpires from all the work that has been rather rapidly surveyed in the preceeding sections is that the available theory of the internal structure of neutron stars seems to provide all the elements needed to account for the accumulated pulsar frequency data within the framework of scenarios in which superfluidity and differential rotation commonly play an essential role. However we are still a long way short of reaching any concensus about the detailed modelling of the many different kinds of behaviour observed in particular cases such as the Crab and Vela pulsars. Before any definitive understanding can be reached it will be necessary to carry out much more work on the technicalities of basic physical processes, particularly those involving electromagnetic effects, which were barely mentioned in the preceeding overview, but that are extremely important for the detailed estimation of important quantities such as the drag to lift ratio, $c_{\mathrm{r}}$.

The remainder of this article will be concerned with just one of the many technical problems that needs to be dealt with before a satisfactorily complete understanding can be achieved. This is the problem of developing an appropriately relativistic treatment of the superfluidity that has been seen to play such an essential role in accounting for cases such as that of Vela, and even the less extreme case of the Crab.

\section{Essentials of relativistic superfluid mechanics}

\subsection{Motivation and background.}

In calculations of global quantities such as the mass and radius of a neutron star with a given baryon number, it has been known since before the earliest pulsar observations in 1968 that a fully relativistic treatment is indispensible for even a minimally acceptable level of accuracy. It is fortunate that quantities such as this [3] can be obtained within the framework of an exactly spherical perfect fluid description for which a fully relativistic treatment is easily applicable and has always been used. This contrasts with what has been done about secondary effects such as precession [50, 27, 51], involving mechanisms such as elasticity (for which a relativistic treatment has long been available [52, 53 but is difficult to apply) and superfluidity 
(for which the relevant macroscopic treatment [54, 55, 56] is relatively new). Even in the relatively tractable context of stationary axisymmetric problems, or in contexts involving the (intrinsically relativistic) effect of gravitational radiation, superfluidity and superconductivity have nearly always been dealt with using a non-relativistic Newtonian, even in relatively recent work [57, 58, 59]

There are two essentially different reasons why it is worthwhile to try to do better. One is of course that a fully relativistic treatment should in principle be more accurate, and will no doubt become necessary for this purpose sooner or later. However in the short run this is not always what is most important, since the errors inherent in the use of a purely Newtonian treatment range typically from a few per cent to a few tens of percent which is not very significant compared with the order of unity (or worse) uncertainties about many of the physical quantities involved. The other kind of reason (which some readers may find surprising) is that for many purposes the use of a relativistic treatment is actually easier. In many cases the advantage of a relativistic treatment is due to the fact that the Lorentz group is in the technical sense semi-simple, whereas the Galilei group unfortunately is not. However, whether or not it is intrinsically simpler, the use of a relativistic treatment will usually be more convenient in practice whenever one wishes to use the commonly appropriate strategy [21, 60, 61] of working in terms of perturbations of the available spherically symmetric perfect fluid neutron star models. This is just because (as remarked above) all the best of these models (the only ones that are commonly taken seriously) are already formulated exclusively in a (general) relativisic framework. The same consideration applies to the perturbations of the relativistic axisymmetric rapidly rotating star models that have recently 62, 63, 64, 65, 66] been a subject of rapid development, particularly in relation to the question of bar mode instabilities that may be significant as a source of gravitational radiation.

The treatment provided here will be limited to the case of scalar (spin 0) models such as are appropriate for the experimentally familiar example of helium - 4 (though not 67] helium - 3) and also for the mesoscopic description of the neutron fluid that (as discussed above) is predicted to interpenetrate the ions in the lower crust of a neutron star, and that is believed [68] to condense as a superfluid in which the relevent bosons are scalar Cooper type pairs of neutrons. For the mesoscopic (intervortex) treatment of the mixed proton neutron superfluid below the crust a scalar description has commonly been employed [18, 69] in a Newtonian treatment, and an analogous relativistic description [70, 71] has recently been made available. However for an exact description of such a mixed proton neutron superfluid, in which it is predicted [68] that the neutrons pair up as bosons of spin 1, it would be necessary to use a more elaborate treatment that has yet to be developed).

What is actually needed for the analysis of large scale effects (such as were considered in the preceeding sections) is not just a mesoscopic treatment of the superfluid on scales small compared with the spacing between interpenetrating ionic nuclei and the vortices where the irrotationality condition breaks down, but a macroscopic average over much larger scales. An appropriate macroscopic theory of the kind that is needed has recently been developed [56] and is described in the final subsections of this article. The treatment presented here differs from the relativistic analogue [54, 55] of the earlier non relativistic description [72] of the averaged effect of vortices in neglecting the small anisotropy due to their effective tension, but instead it includes allowance for what in the long run is likely to be a more important effect, namely the "transfusion" of matter (for the reasons discussed in the previous subsections) between the superfluid constituent and the normal background, which is to be interpreted as representing 
not just thermal excitations (as in ordinary liquid helium - 4) but the entire crust component. A more elaborate treatment would include allowance for anisotropy of the crust constituent which, as noted above, will be cold enough (except very near the surface) to behave as an elastic solid: the way to do this has been indicated elsewhere [73, but such a mixed fluid solid description has not yet been developed in detail, and will not be dealt with in the introductory treatment provided here.

As a preliminary to the construction of model [56] that is actually needed for the macroscopic treatment of neutron star matter, this presentation starts by recapitulating the long well known essentials of the relativistic version of the single constituent kind of superfluid model that is appropriate for the description of Helium 4 at zero temperature, and of of the more recently developed generalisation [74, 75, 76, to a two constituent model (of the kind whose non-relativistic analogue was originally developped by Landau) in which the second constituent represents a gas of phonon excitations.

\subsection{Single constituent perfect fluid models}

Before getting into the specific technicalities of superfluidity, it is worthwhile to start by recapitulating the relevant properties of ordinary barotropic fluid models, which includes the category of single constituent (scalar) superfluid models (representing the zero temperature limit of Landau type 2 constituent models) as the special case in which the vorticity is zero. The vorticity, in this context, is to be interpreted as the meaning the exterior derivative of the relevant momentum covector which will be formally defined below, so the vanishing of the vorticity is the condition for this momentum to be the gradient of a scalar potential, which in the superfluid case is to be understood to be proportional to the phase angle of an underlying bosonic quantum condensate. The qualification that this (zero temperature limit) model is barotropic simply means that there is only one independent state function such as the conserved (e.g. baryon) number density $n$ or the mass density $\rho$ (which are proportional in Newtonian theory but non-linearly related in relativistic theory) on which all the other state functions, such as the pressure $P$ are dependent. The equation of state giving $P$ as a function of $\rho$ will also determine a corresponding speed $c_{\text {I }}$ say, of ordinary "first" sound, that will be given by the familiar formula

$$
c_{\mathrm{I}}^{2}=d P / d \rho,
$$

and that must be subluminal, $c_{\mathrm{I}}^{2} \leq c^{2}$ (where $c$ is the speed of light) in order for the usual causality requirement to be respected.

Before proceeding it is desirable to recall the essential elements of the relativistic kinematics and dynamics that will be required. This is particularly necessary in view of the regretable tradition in non-relativistic fluid theory - and particularly in non relativistic superfluid theory - of obscuring the essential distinction between velocity (which formally belongs in a tangent bundle) and momentum (which formally belongs in a cotangent bundle) despite the fact that the distinction is generally respected in other branches of non-relativistic condensed matter theory, such as solid state physics, where the possibility of non-alignment between the 3-velocity $v^{a}$, and the effective 3 -momentum $p_{a}$ of an electron travelling in a metallic lattice is well known. Failure to distinguish between contravariant entities (with "upstairs" indices) such as the velocity $v^{a}$ and covariant entities (with "downstairs" indices) such as the momentum $p_{a}$ is something that one can get away with in a non-relativistic treatment only 
at a price that includes restriction to strictly Cartesian (rather than for example cylindrical or comoving) coordinates.

In a relativistic treatment, even using coordinates $x^{\mu} \leftrightarrow\left\{t, x^{a}\right\}$ of Minkowski type, with a flat spacetime metric $g_{\mu \nu}$ whose components are of the fixed standard form $\operatorname{diag}\left\{-c^{2}, 1,1,1\right\}$, the necessity of distinguishing between raised and lowered indices is inescapable. Thus for a trajectory parametrised by proper time $\tau$, the correponding unit tangent vector

$$
u^{\mu}=\frac{d x^{\mu}}{d \tau}
$$

is automatically, by construction, a contravariant vector: its space components, $u^{a}=\gamma v^{a}$ with $\gamma=\left(1-v^{2} / c^{2}\right)^{-1 / 2}$ will be unaffected by the index lowering operation $u^{\mu} \mapsto u_{\mu}=$ $g_{\mu \nu} u^{\nu}$, but its time component $u^{0}=d t / d \tau=\gamma$ will differ in sign from the corresponding component $u_{0}=-\gamma c^{2}$ of the associated covector $u_{\mu}$. On the other hand the 3-momentum $p_{a}$ and energy $E$ determine a 4-momentum covector $\mu_{\nu}$ with components $\pi_{a}=p_{a}, \mu_{0}=-E$ that are intrinsically covariant. The covariant nature of the momentum can be seen from the way it is introduced by the defining equation,

$$
\mu_{\nu}=\frac{\partial L}{\partial u^{\nu}}
$$

in terms of the relevant position and velocity dependent Lagrangian function $L$, from which the corresponding equation of motion is obtained in the well known form

$$
\frac{d \mu_{\nu}}{d \tau}=\frac{\partial L}{\partial x^{\nu}}
$$

In the case of a free particle trajectory, and more generally for fluid flow trajectories in a model of the simple barotropic kind that is relevant in the zero temperature limit, the Lagrangian function will have the familiar standard form

$$
L=\frac{1}{2} \mu g_{\mu \nu} u^{\mu} u^{\nu}-\frac{1}{2} \mu c^{2},
$$

in which (unlike what is needed for more complicated chemically inhomogeneous models 77 , 73) it is the same scalar spacetime field $\mu$ that plays the role of mass in the first term and that provides the potential energy contribution in the second term. The momentum will thus be given by the simple proportionality relation

$$
\mu_{\nu}=\mu u_{\nu}
$$

so that one obtains the expressions $E=\gamma \mu c^{2}, p_{a}=\mu \gamma v_{a}$, in which the field $\mu$ is interpretable as the relevant effective mass.

In the case of a free particle model, the effective mass $\mu$ will of course just be a constant, $\mu=m$. This means that if, as we have been supposing so far, the metric $g_{\mu \nu}$ is that of flat Minkowski type, the resulting free particle trajectories will be obtainable trivially as straight lines. However the covarant form of the equations (47) to (51) means that they will still be valid for less trivial cases for which, instead of being flat, the metric $g_{\mu \nu}$ is postulated to have a variable form in order to represent the effect of a gravitational field, such as that of a Kerr black hole (for which, as I showed in detail in a much earlier Les Houches school [78], the resulting non trivial geodesic equations still turn out to be exactly integrable). 
In the case of the simple "barotropic" perfect fluid models with which we shall be concerned here, the effective mass field $\mu$ will be generically non-uniform. In these models the equation of state giving the pressure $P$ as a function of the mass density $\rho$ can most conveniently be specified by first giving $\rho$ in terms of the corresponding conserved number density $n$ by an expression that will be decomposible in the form

$$
\rho=m n+\frac{\epsilon}{c^{2}}
$$

in which $m$ is a fixed "rest mass" characterising the kind of particle (e.g. a Cooper type neutron pair) under consideration, while $\epsilon$ represents an extra compression energy contribution. The pressure will then be obtainable using the well known formula

$$
P=(n \mu-\rho) c^{2},
$$

in which the effective dynamical mass is defined by

$$
\mu=\frac{d \rho}{d n}=m+\frac{1}{c^{2}} \frac{d \epsilon}{d n} .
$$

It is this same quantity $\mu$ (sometimes known as the "specific enthalpy") that is to be taken as the effective mass function appearing in the specification (50) of the relevant Lagrangian function (on what is formally identifiable as the tangent bundle of the spacetime manifold).

When one is dealing not just with a single particle trajectory but a spacefilling fluid flow, it is possible and for many purposes desirable to convert the Lagrangian dynamical equation (49) from particle evolution equation to equivalent field evolution equations [77, 73]. Since the momentum covector $\mu_{\nu}$ will be obtained as a field over spacetime, it will have a well defined gradient tensor $\nabla_{\rho} \mu_{\nu}$ that can be used to rewrite the right hand side of (49) in the form $d \mu_{\nu} / d \tau=u^{\rho} \nabla_{\rho} \mu_{\nu}$. Since the value of the Lagrangian will also be obtained as a scalar spacetime field $L$, it will also have a well defined gradient which will evidently be given by an expression of the form

$$
\nabla_{\nu} L=\frac{\partial L}{\partial x^{\nu}}+\frac{\partial L}{\partial \mu_{\rho}} \nabla_{\nu} \mu_{\rho}
$$

We can thereby rewrite the Lagrangian dynamical equation (49) as a field equation of the form

$$
u^{\rho} \nabla_{\rho} \mu_{\nu}+\mu_{\rho} \nabla_{\nu} u^{\rho}=\nabla_{\nu} L
$$

An alternative approach is of course to start from the corresponding Hamiltonian function, as obtained in terms of the position and momentum variables (so that formally it should be considered as a function on the spacetime cotangent bundle) via the Legendre transformation

$$
H=\mu_{\nu} u^{\nu}-L .
$$

In this approach the velocity vector is recovered using the formula

$$
\frac{d x^{\mu}}{d \tau}=\frac{\partial H}{\partial \mu_{\nu}},
$$

and the associated dynamical equation takes the form

$$
\frac{d \mu_{\nu}}{d \tau}=-\frac{\partial H}{\partial x^{\nu}} .
$$


The consideration that we are concerned not just with a single trajectory but with a spacefilling fluid means that, as in the case of the preceeding Lagrangian equations, so in a similar way this familiar Hamiltonian dynamical equation can also be converted to a field equation which takes the form

$$
2 u^{\rho} \nabla_{[\rho} \mu_{\nu]}=-\nabla_{\nu} H
$$

with the usual convention that square brackets are used to indicate index antisymmetrisation. On contraction with $u^{\nu}$ the left hand side will evidently go out, leaving the condition

$$
u^{\nu} \nabla_{\nu} H=0
$$

expressing the conservation of the value of the Hamiltonian allong the flow lines.

The actual form of the Hamiltonian function that is obtained from the simple barotropic kind of Lagrangian function (50) with which we are concerned will evidently be given by

$$
H=\frac{1}{2 \mu} g^{\nu \rho} \mu_{\nu} \mu_{\rho}+\frac{\mu c^{2}}{2},
$$

in which it is again the same scalar spacetime field $\mu$ that plays the role of mass in the first term and that provides potential energy contribution in the second term.

In order to ensure the proper time normalisation for the parameter $\tau$ the equations of motion (in whichever of the four equivalent forms (49), (55), (58), (59) may be preferred) are to be solved subject to the constraint that the numerical value of the Hamiltonian should vanish,

$$
H=0,
$$

initially, and hence also by (60) at all other times. This is evidently equivalent to imposing the standard normalisation condition

$$
u^{\mu} u_{\mu}=-c^{2}
$$

on the velocity four vector. In more general "non-barotropic" systems, such as are needed for some purposes, the Hamiltonian may be constrained in a non uniform manner [77, 73] so that the term on the right of (59) will be non zero, but in the simpler systems that suffice for our present purpose the restraint (62) ensures that this final term will drop out, leaving a Hamiltonian equation that takes the very elegant and convenient form

$$
u^{\nu} w_{\nu \rho}=0 \text {. }
$$

in terms of the relativistic vorticity tensor that is defined as the antisymmetrised ("exterior") derivative of the momentum covector, i.e.

$$
w_{\nu \rho}=2 \nabla_{[\nu} \mu_{\rho]} .
$$

It is an evident consequence (and, as discussed in greated detail in the above cited Les Houches notes [55], would still be true even if (62) were not satisfied) that if $w_{\mu \nu}$ is zero initially it will remain zero throughout the flow, which in this case will be describable as "irrotational".

In cases for which the vorticity is non-zero, the "barotropic" dynamical equation (64) is interpretable as requiring the flow vector $u^{\mu}$ to be a zero eigenvalue eigenvector of the vorticity 
tensor $w_{\mu \nu}$, which is evidently possible only if its determinant vanishes, a requirement that is expressible as the degeneracy condition

$$
w_{\mu[\nu} w_{\rho \sigma]}=0 .
$$

Since the the possibility of it having matrix rank 4 is thus excluded, it follows that unless it actually vanishes the vorticity tensor must have rank 2 (since an antisymmetric matrix can never have odd integer rank). This means that the flow vector $u^{\mu}$ is just a particular case within a whole 2-dimensional tangent subspace of zero eigenvalue vorticity eigenvectors, which (by a well known theorem of differential form theory) will mesh together to form well defined vorticity 2 -surfaces as a consequence of the Poincaré closure property,

$$
\nabla_{[\mu} w_{\nu \rho]}=0
$$

that follows from the definition (65).

Although it has long been well known to specialists [82], the simple form (64) of what is interpretable just as the relativistic version of the classical Euler equation is still not as widely familiar as it ought to be, perhaps because its Hamiltonian interpretation was not recognised until relatively recently [77, 73]. It does not constitute by itself the complete set of dynamical equations of motion for the perfect fluid, but must be supplemented by a particle conservation equation of the usual form for the particle number current

$$
n^{\nu}=n u^{\nu},
$$

which must of course satisfy the condition

$$
\nabla_{\nu} n^{\nu}=0
$$

A much more widely known, but for computational purposes (particularly in curved spacetime) less useful form of the perfect fluid dynamical equations is to express them in terms of the stress momentum energy density tensor, which is given in terms of the mass density $\rho$ and the pressure $P$ by

$$
T^{\mu \nu}=\left(\rho+\frac{P}{c^{2}}\right) u^{\mu} u^{\nu}+P g^{\mu \nu},
$$

and which must satisfy a so called conservation law of the standard form

$$
\nabla_{\nu} T^{\mu \nu}=0
$$

Although it is conveniently succinct, a disadvantage of this traditional formulation is that it is directly interpretable as a law of conservation of momentum and energy in the strict sense only in the case of a flat (Minkowski type) spacetime, but not in a curved background such as that of a neutron star. The possibility in the barotropic case (i.e. when $P$ is a function only of $\rho$ ) of decomposing the combined set of dynamical equations (70) as the combination of the convergence condition (69) (obtained by contracting (71) with $u_{\mu}$ ) and the relativistic Euler equation (64), which can be written out more explicitly as

$$
n^{\nu} \nabla_{[\nu} \mu_{\rho]}=0
$$

has the advantage that these are interpretable as genuine conservation laws - for particle number flux and vorticity respectively - even in an arbitrarily curved spacetime background. 


\section{Single constituent superfluid models}

The simplest superfluid models, namely those pertaining to the zero temperature limit, are just ordinary perfect fluid models subject to the restraint of irrotationality, with a momentum covector given as the gradient

$$
\mu_{\nu}=\nabla_{\nu} S
$$

of a scalar field $S$. This scalar field is to interpreted as being proportional to the angle of the mesoscopic phase factor, $\mathrm{e}^{i \phi}$ say, of an underlying scalar bosonic condensate, in which the phase angle $\phi$ is given according to the usual correspondence principle by

$$
\phi=S / \hbar \text {. }
$$

In the most familiar application the bosons are Helium- 4 atoms, while between the ions of a neutron star crust below the neutron drip transition they will be Cooper type neutron pairs. (However a less simple description is not sufficient for the spin 1 neutron pairs below the base of the crust, nor in the even more complicated, though experimentally accessible, case [67] of Helium-3, for which a relativistic description is still not available).

In a multiconnected configuration of a classical irrotational fluid the Jacobi action field $S$ obtained from (73) might have an arbitrary periodicity, but in a superfluid there will be a $\mathrm{U}(1)$ periodicity quantisation requirement that the periodicity of the phase angle $\phi$ should be a multiple of $2 \pi$, and thus that the periodicity of the Jacobi action $S$ should be a multiple of $2 \pi \hbar$. The simplest configuration for any such superfluid is a uniform stationary state in a flat Minkowski background, for which the phase will have the standard plane wave form

$$
S / \hbar=k_{a} x^{a}-\omega t,
$$

from which one obtains the correspondence $\mu_{\nu} \leftrightarrow\left\{-\hbar \omega, \hbar k_{a}\right\}$, which means that the effective energy per particle will be given by $E=\gamma \mu c^{2}=\hbar \omega$ and that the 3-momentum will be given by $p_{a}=\mu \gamma v_{a}=\hbar k_{a}$.

It is to be remarked that for ordinary timelike superfluid particle trajectories the corresponding phase speed $\omega / k$ of the wave characterised by (75) will always be superluminal - a fact of which people working with laboratory Helium-4 tend to be blissfully unaware, and can usually safely ignore, since what matters for most practical purposes is not the phase speed but the group velocity of perturbation wave packets.

In the irrotational case characterised by $(\sqrt{73})$ the Euler equation (64) is satisfied automatically, so the only dynamical equation that remains is (69). When the phase scalar is subject to a small perturbation, $\delta \phi=\varphi$ say, it can be seen that the corresponding perturbation of the conservation law (69) provides a wave equation of the form

$$
\widetilde{\square} \varphi=0,
$$

in which $\widetilde{\square}$ is a modified Dalembertian type operator that is constructed from an appropriately modified space-time metric tensor $\widetilde{g}^{\mu \nu}$ in the same way that the ordinary Dalembertian operator $\square \equiv \nabla^{\mu} \nabla_{\mu}$ is constructed from the ordinary spacetime metric tensor $g^{\mu \nu}$. The appropriately modified spacetime metric, namely the relativistic version of what is known in the context of Newtonian fluid [79, 80] and superfluid [81] mechanics as the Unruh metric, can be read out in terms of the light speed $c$ and the (first) sound speed $c_{\mathrm{I}}$ given by (46) as

$$
\widetilde{g}^{\mu \nu}=\frac{\mu}{n}\left(c_{\mathrm{I}} \gamma^{\mu \nu}-c_{\mathrm{I}}^{-1} u^{\mu} u^{\nu}\right),
$$


where $\gamma^{\mu \nu}$ is the spacially projected (positive indefinite) part of the ordinary space time metric, as defined by

$$
\gamma^{\mu \nu}=g^{\mu \nu}+c^{-2} u^{\mu} u^{\nu} .
$$

The quantum excitations of the linearised perturbation field $\varphi$ governed by (76) are what are known as phonons. For such excitations the phase speed and the group velocity are the same, both being given with respect to the unperturbed background by the ordinary ("first") soundspeed, $c_{\mathrm{I}}$, as given by (46), which will of course be subluminal. Phonons do nevertheless have a tachyonic aspect of their own, because the fact that their phase speed is subluminal automatically implies that they have a 4-momentum covector that is spacelike, in contrast with that of a ordinary fluid or superfluid particle which is timelike. This means that whereas the effective energy $E$ of an ordinary fluid or superfluid particle is always positive, the effective energy $E$ of a phonon may be positive or negative, depending on whether the frame of reference with respect to which it is measured is moving subsonically or supersonically. The well known implication is that if the superfluid is in contact with a supersonically moving boundary there will inevitably be an instability giving rise to dissipative phonon creation.

Given a dynamical system, one of the first things any physicist is inclined to ask is whether it is derivable from a Lagrangian type variation principle. We have already seen in the previous sections that (64) by itself is obtainable from Lagrangian equations of motion for the individual trajectories, which are of course obtainable from a one dimensional action integral of the form $\int L d \tau$ with $L$ as given by (50). The question to be adressed now is how to obtain the complete set of dynamical equations (71), including (64) as well as (69), from an action integral over the 4-dimensional background manifold $\mathcal{S}^{(4)}$ of the form

$$
\mathcal{I}=\int \mathcal{L} d \mathcal{S}^{(4)}, \quad d \mathcal{S}^{(4)}=\frac{\|g\|^{1 / 2}}{c} d^{4} x
$$

for some suitable scalar Lagrangian functional $\mathcal{L}$.

There are several available procedures for doing this for a generic perfect fluid with rotation, involving radically different choices of the independent variables to be varied: although they are all ultimately equivalent "on shell" the "off shell" bundles over which the variations are taken differ not only in structure but even in dimension. These methods notably include the worldline variation procedure (the most economical from a dimensional point of view) developed by Taub 83], and the Clebsch type variation proceedure developed by Schutz 84], as well as the more recently developed Kalb-Ramond type method [85, 55] that has been specifically designed for generalisation [54] to allow for the anisotropy arising from the averaged effect of vortex tension in the treatment of superfluidity at a macroscopic level. None of these various methods is sufficiently simple to have become widely popular.

The problem is much easier to deal with if, to start off with, one restricts oneself to the purely irrotational case characterised by (73), which is all that is needed for the description of zero temperature superfluidity at a mesoscopic level. In this case a very simple and well known procedure is available. In this procedure, the independent variable is taken to be just the Jacobi action $S$, or equivalently in a superfluid context, the phase $\varphi$ as given by (74), and the action is simply taken to be the pressure $\mathrm{P}$ expressed as a function of the effective mass $\mu$, with the latter constructed as proportional to the amplitude of the 4-momentum, according to the prescription

$$
\mu^{2} c^{2}=-\mu_{\nu} \mu^{\nu}
$$


with the 4-momentum itself given by the relation (73) that applies in the irrotational case, i.e.

$$
\mu_{\nu}=\hbar \nabla_{\nu} \phi
$$

Thus setting

$$
\mathcal{L}=P,
$$

and using the standard pressure variation formula

$$
\delta P=c^{2} n \delta \mu,
$$

one sees that the required variation of the Lagrangian will be given by

$$
\delta \mathcal{L}=-n^{\nu} \delta \mu_{\nu}=-\hbar n^{\nu} \nabla_{\nu}(\delta \phi) .
$$

Demanding that the action integral $(79)$ be invariant with respect to infinitesimal variations of $\varphi=\delta \phi$ then evidently leads to the required conservation law (69).

It is to be noted that this variational principle can be reformulated in terms of an independently variable auxiliary field amplitude $\Phi$ and an appropriately constructed potential function $V\{\Phi\}$ as a function of which the action takes the desirably fashionable form

$$
\mathcal{L}=-\frac{\hbar^{2}}{2} \Phi^{2}\left(\nabla_{\nu} \phi\right) \nabla^{\nu} \phi-V\{\Phi\}
$$

which is interpretable as the classical limit of a generalised Landau Ginzburg type model. In this formulation, as discussed in greater detail elsewhere 85, 55, the auxiliary amplitude is to be identified as being given by the formula

$$
\Phi=\frac{n}{\sqrt{\rho+P / c^{2}}}=\left(\frac{n}{\mu}\right)^{1 / 2}
$$

while the prescription for the corresponding potential energy density function is that it should be given by

$$
V=\frac{\rho c^{2}-P}{2} .
$$

Having evaluated $V$ as a function of $\Phi$ one can recover the effective mass $\mu$, number density $n$, mass density $\rho$ and pressure $P$ of the fluid using the formulae

$$
\mu^{2}=\frac{1}{c^{2} \Phi} \frac{d V}{d \Phi}, \quad n=\Phi^{2} \mu
$$

and

$$
\rho=\frac{1}{2} \Phi^{2} \mu^{2}+\frac{V}{c^{2}}, \quad P=\frac{1}{2} \Phi^{2} \mu^{2} c^{2}-V,
$$

which are derivable from (53) and (54). It is to be remarked that the covariant inverse of the generalised Unruh tensor (77) is expressible in this notation as

$$
\widetilde{g}_{\mu \nu}^{-1}=\Phi^{2}\left(c_{\mathrm{I}}^{-1} \gamma_{\mu \nu}-c_{\mathrm{I}} c^{-2} u_{\mu} u_{\nu}\right) .
$$

A particularly noteworthy example is the conformally invariant special case 55 characterised by a potential function that is homogeniously quartic, $V \propto \Phi^{4}$, which is what is obtained for a radiation gas type equation of state of the familiar form $P=\rho c^{2} / 3$, and for which the (first) sound speed is given by $c_{\mathrm{I}}^{2}=c^{2} / 3$. 


\section{Landau type 2-constituent superfluid models.}

As an intermediate step between the very simple single constituent superfluid models described in the previous section and the more elaborate models needed in the context of neutron stars, the purpose of this section is to describe the relativistic version of the category of non dissipative 2-constituent superfluid that was originally developed by Landau for the description of ordinary superfluid Helium-4 at non-zero temperature. As well as the relevant conserved particle number current $n^{\mu}$ (representing the flux of Helium atoms in that particular application) such a model involves another independently conserved current vector, $s^{\mu}$ say, representing the flux of entropy. In the single constituent case characterised by the variation rule (84) we saw how the current vector $n^{\nu}$ was associated with a dynamically conjugate covector $\mu_{\nu}$ that is interpretable as representing the effective mean 4-momentum per particle. In a similar way in a 2-constituent model the second current vector $s^{\nu}$ will be analogously associated with its own dynamically conjugate 4-momentum covector $\Theta_{\nu}$.

The earliest presentations of the generic category of non-dissipative 2-constituent superfluid were on one hand a generalisation [74] of the relativistic Clebsch formulation [84 based on the variation of a generalised pressure function $\Psi$ depending on the 4-momentum covectors $\mu_{\nu}$ and $\Theta_{\nu}$ according to the partial differentiation rule

$$
d \Psi=-n^{\nu} d \mu_{\nu}-s^{\nu} d \Theta_{\nu},
$$

and on the other hand a generalisation [86, 73] of the world line variational formulation due to Taub 83] based on the variation of a master function $\Lambda$ depending on the currents $n^{\mu}$ and $s^{\nu}$ according to the partial differentiation rule

$$
d \Lambda=\mu_{\nu} d n^{\nu}+\Theta_{\nu} d s^{\nu} .
$$

Although they were originally developed independently these alternative formulations were subsequently shown to be equivalent to each other and to an intermediate crossbred version [75] based on a Lagrangian density

$$
\mathcal{L}=\Psi+s^{\nu} \Theta_{\nu}=\Lambda-n^{\mu} \mu_{\nu}
$$

depending on the particle 4-momentum covector $\mu_{\nu}$ and the entropy current $s^{\nu}$ according to the partial differentiation rule

$$
d \mathcal{L}=\Theta_{\nu} d s^{\nu}-n^{\nu} d \mu_{\nu}
$$

All of these variational formulations are subject to the complication that the allowable field variations are not free but must be suitably constrained to avoid giving overdetermined field equations. Although it violates the symmetry between the two kinds of conserved current $n^{\nu}$ and $s^{\nu}$ that are involved, the crossbred formulation characterised by (94) is the one that allows the simplest specification of the constraints required to get the appropriate dynamical equations for the superfluid case. In this formulation [75] the constraint on the particle 4momentum covector is simply that it should have the same phase gradient form (73) as in the zero temperature limit in which the entropy constituent is absent, namely

$$
\mu_{\nu}=\hbar \nabla_{\nu} \phi
$$

The corresponding constraint on the current vector $s^{\nu}$ of the "normal" constituent is the not quite so simple Taub type requirement that its variation should be determined by the 
displacement of the flow lines generated by an arbitrary vector field $\zeta^{\nu}$ say, which means [73] that it must have the form

$$
d s^{\nu}=\zeta^{\rho} \nabla_{\rho} s^{\nu}-s^{\nu} \nabla_{\rho} \zeta^{\nu}+s^{\nu} \nabla_{\rho} \zeta^{\nu}
$$

whose derivation is obtainable by a procedure that will be explained more explicitly in the next Section. Demanding invariance of the volume integral of $\mathcal{L}$ with respect to infinitesimal local variations of the phase variable $\phi$ then gives the usual particle conservation law in the same form (69) as for the single constituent limit, while demanding invariance for an arbitrary local displacement field $\zeta$ gives not only the analogous entropy conservation law

$$
\nabla_{\nu} s^{\nu}=0
$$

but also the dynamical equation,

$$
s^{\nu} \nabla_{[\nu} \Theta_{\rho]}=0,
$$

that governs the evolution of the thermal 4-momentum covector in a manner analogous to that whereby the relativistic Euler equation (72) governs the evolution of the momentum covector in an ordinary perfect fluid. These dynamical equations entail (but unlike the single constituent case are not entirely contained in) an energy momentum pseudo-conservation law of the usual form (71) for a stress-momentum-energy density tensor that can be written in the form

$$
T_{\rho}^{\nu}=n^{\nu} \mu_{\rho}+s^{\nu} \Theta_{\rho}+\Psi g_{\rho}^{\nu}
$$

which will in fact (although it is not obvious in this particular expression) be automatically symmetric, $T^{[\nu \rho]}=0$.

The category of models characterised by the preceeding specifications for various conceivable forms of the equation of state specifying $\mathcal{L}$ as a scalar function of $\mu_{\nu}$ and $s^{\nu}$ is very large. The use of what is interpretable [87] as a special subcategory therein, on the basis of a particular kind of separation ansatz, was proposed in early work of Israel [88] and Dixon [89] and has been advocated more recently by Olsen [90]. Unfortunately however, the simplification provided by the Israel Dixon ansatz (effectively the relativistic generalisation of the obsolete Tisza-London theory that was superceded by that of Landau) is incompatible with the kind of equation of state that is needed for even a minimally realistic treatment of a real superfluid.

A satisfactory treatment of what goes on at temperatures high enough for non-linear "roton" type excitations to be important is not yet available, but in the low temperature "cool" regime, in which only linear "phonon" type excitations are important, it is not difficult to provide a straightforward analytic derivation of the kind of equation of state that is appropriate. Following the lines developed in a non - relativistic context by Landau himself [91] the relativistic version of the approriate "cool" equation of state has recently been derived [76] by considering perturbations of the single constituent model - with equation of state specified as a pressure function, $P\{\mu\}$ - that describes the relevant zero temperature limit. The result is obtained in an analytically explicit form that (despite the fact that it is not of the separable Israel Dixon kind) can be given a very simple expression in terms of what we referred to as the "sonic" metric, which is specifiable by the conformal relation

$$
\mathcal{G}^{\rho \sigma}=\Phi^{2} c_{\mathrm{I}}^{-1} \widetilde{g}^{\rho \sigma}
$$

in terms the Unruh phonon metric (77) that is associated with the relevant zero temperature limit state as specified by the relevant momentum covector $\mu_{\nu}$, which by (80) determines the 
relevant value of the scalar $\mu$ and hence (via the zero temperature equation of state, using the formalism of Section 2.2) also of the relevant phonon speed $c_{\mathrm{I}}$ and field amplitude $\Phi$. While the Unruh metric is more convenient for many purposes, the advantage of the conformal modification we have used, namely

$$
\mathcal{G}^{\rho \sigma}=g^{\rho \sigma}+\left(c^{-2}-c_{\mathrm{I}}^{-2}\right) u^{\rho} u^{\sigma},
$$

is that its spacially projected part agrees with that of the ordinary space metric, from which it differs only in the measurement of time.

The result that is obtained [76] is given by a Lagrangian of the form

$$
\mathcal{L}=P-3 \psi
$$

in which the deviation from the zero pressure limit value $P\{\mu\}$ is given as a function not just of the particle 4-momentum covector $\mu_{\nu}$ but also of the entropy flux $s^{\nu}$ (postulated to be sufficiently weak to be constituted only of phonons) by the formula

$$
\psi=\frac{\tilde{\hbar}}{3} c_{\mathrm{I}}^{-1 / 3}\left|\mathcal{G}_{\rho \sigma}^{-1} s^{\rho} s^{\sigma}\right|^{2 / 3},
$$

where $\tilde{\hbar}$ is identifiable to a very good approximation with the usual Dirac-Planck constant $\hbar$, its exact value being given by

$$
\tilde{\hbar}=\frac{9}{4 \pi}\left(\frac{5 \pi}{6}\right)^{1 / 3} \simeq 0.99 \hbar .
$$

This is equivalent to taking the generalised pressure function to be

$$
\Psi=P+\psi,
$$

with

$$
\psi=\frac{c_{\mathrm{I}}}{4}\left(\frac{3}{4 \hbar}\right)^{3}\left(\mathcal{G}^{\rho \sigma} \Theta_{\rho} \Theta_{\sigma}\right)^{2},
$$

in which the effective thermal 4-momentum per unit of entropy is given (according to the partial differentiation formula (94) by

$$
\Theta_{\rho}=\frac{4 \tilde{\hbar}}{3}\left|c_{\mathrm{I}} \mathcal{G}_{\mu \nu}^{-1} s^{\mu} s^{\nu}\right|^{-1 / 3} \mathcal{G}_{\rho \sigma}^{-1} s^{\sigma},
$$

with

$$
\mathcal{G}_{\rho \sigma}^{-1}=g_{\rho \sigma}+\left(1-\frac{c_{\mathrm{I}}^{2}}{c^{2}}\right)\left|\mu^{\nu} \mu_{\nu}\right|^{-1} \mu_{\rho} \mu_{\sigma}
$$

An concrete illustration, allowing the explicit evaluation of the relevant quantities, is provided by the polytropic case, as characterised by a (single consitituent) equation of state giving the mass density $\rho$ as a function of the number density $n$ in terms of a fixed ("rest") mas per particle $m$, a scale constant $\kappa$ and a fixed dimensionless index $\gamma$ in the form

$$
\rho=m n+\kappa n^{\gamma} \Leftrightarrow \mu=m+\kappa \gamma n^{\gamma-1},
$$

which corresponds to taking the pressure to be given by

$$
P=\kappa c^{2}(\gamma-1) n^{\gamma}=\kappa c^{2}(\gamma-1)\left(\frac{\mu-m}{\kappa \gamma}\right)^{\gamma /(\gamma-1)},
$$

while the corresponding sound speed will be given (independently of $\kappa$ ) by

$$
c_{\mathrm{I}}^{2}=(\gamma-1)\left(1-\frac{m}{\mu}\right) c^{2} .
$$




\section{Non-conservative model with transfusion and vortex drag}

Although the Landau type of model described in the previous section has been found to be very effective for the description of liquid Helium-4 under laboratory conditions, it is not of much use for direct application in neutron star matter because the thermal effects it allows for will in general be less important than other complications whose treatment will require the use of more elaborate models whose relativistic versions are still at a relatively early stage of development and will not be presented here. The most important of these complications, whose treatment in a relativistic framework has been the subject of preliminary work that is discussed elsewhere, are due to the effect of the protons that will be present, either in ionic nuclei that are responsible for the elastic solid behavior [73] of the crust, or as a dissolved superfluid [70, 71] at deeper levels. Another complication that is relevant for the macroscopic treatment of a neutron star is the necessity of averaging over an Abrikosov type lattice of quantised vortices (that must be roughly aligned with the rotation axis of the star) whose effective tension entails deviations [54, 55] from perfect fluid isotropy.

Like the thermal effect discussed in the preceding section, these various complications can all be provisionally set aside as perturbations to be incorporated at a later stage in a systematic approach whose first stage requires the use only of a relatively crude description in which, except for the superfluid neutrons with baryon number current vector $n_{\mathrm{n}}^{\nu}$ all the other constituents, meaning mainly protons and electrons, move together with the entropy as a single "normal" constituent with baryon number current $n_{\mathrm{c}}^{\nu}$. Whereas the anisotropy arising from vortex tension [54, 55] is relatively unimportant, a major role in the long term evolution of the star is likely to be played by the static pinning or dynamical drag forces exerted on the vortices by the composite "normal" background constituent. Another effect that is of importance in the long run is that of "transfusion" whereby - due to the subduction resulting from the drift mechanism whose effect is roughly described by $(39)$ or $(40)$ - the superfluid neutron contribution $n_{\mathrm{n}}^{\nu}$ to the baryon current may undergoes transformation (via weak beta decay type processes) to the "normal" (essentially protonic) consituent, and vice versa, so that only the total baryon current

$$
n_{\mathrm{b}}=n_{\mathrm{n}}^{\nu}+n_{\mathrm{c}}^{\nu}
$$

remains locally conserved throughout:

$$
\nabla_{\nu} n_{\mathrm{b}}^{\nu}=0
$$

The kind of (non-conservative) 2-constituent model needed for this purpose is obtainable as a generalisation of the kind of (conservative) 2-constituent superfluid model discussed in the preceding section, starting from the formulation in terms of a master function $\Lambda$ in which the currents (not momenta) are taken as the independent (but not entirely free) variables.

In a transfusive model of the type set up here, the "normal" constituent is not entirely dependent on (though it does include) entropy, so that it is present even at zero temperature: the primary role of this non - superfluid constituent is to represent the fraction of the baryonic material of the neutron star that is not included in the neutron superfluid, as well as the degenerate electron gas that will be present to neutralise the charge density resulting from the fact that some of these baryons will have the form of protons rather than neutrons. In the solid "crust" layers of a neutron star the protons will be concentrated together with a 
certain fraction of the neutrons in discrete nuclear type ions, which at the relatively moderate temperatures that are expected to apply will form a solid lattice. In the upper crust the "normal" constituent consisting of the ionic lattice and the degenerate electrons will include everything, but in the lower crust (at densities above about $10^{11} \mathrm{gm} / \mathrm{cm}^{3}$ ) the crust will be interpenetrated by an independently moving neutron superfluid. What we refer to as "transfusion" occurs when compression takes place so that the ionic constituent undergoes a fusion process whereby neutrons are released in the form of newly created superfluid matter, or conversely, when relaxation of the pressure allows excess neutrons to be reabsorbed into the ions.

A more elaborate treatment would specifically allow for the expectation that the protons would form an independently conducting superfluid of their own at very high densities, whereas they will combine with some of the neutrons at intermediate densities, and with all of the neutrons at low densities, to form discrete ions which will tend to crystalise to form a possibly anisotropic lattice. What matters for our present purpose is that regardless of its detailed constitution, all this "normal" matter will in effect be strongly self coupled [19] by short range electromagnetic interactions so that its movement will be describable to a very good approximation as that of a single fluid with a well defined 4 -velocity, $u_{\mathrm{c}}{ }^{\mu}$ say, the only independent motion being that of the (electromagnetically neutral) neutron superfluid with velocity ${u_{\mathrm{n}}}^{\mu}$ say. The latter will specify the direction of the part of the baryon current,

$$
n_{\mathrm{n}}{ }^{\mu}=n_{\mathrm{n}} u_{\mathrm{n}}{ }^{\mu}
$$

carried by the neutron superfluid, while the "normal" matter velocity specifies the direction of the remaining collectively comoving part,

$$
n_{\mathrm{c}}{ }^{\mu}=n_{\mathrm{c}} u_{\mathrm{c}}{ }^{\mu}
$$

of the baryon current.

At densities below the "neutron drip" transition at about $10^{11} \mathrm{gm} / \mathrm{cm}^{3}$, the "normal" collectively comoving constituent $n_{\mathrm{c}}{ }^{\mu}$ will of course be identifiable with the total, $n_{\mathrm{b}}{ }^{\mu}$. The reason why the remaining free neutron part $n_{\mathrm{n}}{ }^{\mu}$ - which will always be present at higher densities - is presumed to be in a state of superfluidity is that the relevant condensation temperature, below which the neutrons form bosonic condensate of Cooper type pairs is estimated 92 to be at least of the order of $10^{9} \mathrm{~K}$, while it is expected that a newly formed neutron star will drop substantially below this temperature within a few months [93]. At such comparatively low temperatures the corresponding entropy current $s^{\mu}$ say will not play a very important dynamical role, but for the sake of exact internal consistency it will be allowed for in the model set up here, in which it will be taken for granted that it forms part of the "normal" collectively comoving constituent so that it will have the form

$$
s^{\mu}=s u_{\mathrm{c}}^{\mu} .
$$

Under conditions of sufficiently slow convection, the transfer needs not involve significant dissipation, so the process should be describable by a Lagrangian scalar, $\Lambda$ say, that will depend just on the currents introduced above, of which the independent components are given just by the vectors $n_{\mathrm{c}}^{\mu}$ and $n_{\mathrm{n}}^{\mu}$ and the scalar $s$. As a first approximation (whose accuracy in the various relevant density regimes is a subject that needs much further investigation) one might suppose that the Lagrangian separates in the form $\Lambda=-\rho_{\mathrm{c}} c^{2}-\rho_{\mathrm{n}} c^{2}$ in which $\rho_{\mathrm{c}}$ is a 
mass density depending only on $s$ and $n_{\mathrm{c}}$, while $\rho_{\mathrm{n}}$ is an another energy mass depending only on $n_{\mathrm{n}}$, but we shall not invoke such a postulate here, i.e. we allow for the likelihood that the properties of "normal" constituent will be affected by the presence of the superfluid constituent and vice versa, which means that there will be an entrainment effect [94, 18, 19, 95], whereby for example the velocity of the superfluid neutron current will no longer be parallel to the corresponding momentum. (As an alternative to the more suitable term "entrainment" this mechanism is sometimes referred to in the litterature as "drag", which is misleading because entrainment is a purely conservative, entirely non-dissipative effect, whereas the usual kinds of drag in physics, and in particular the kind of drag to be discussed below, are essentially dissipative processes.)

If we adopted the (gas type) description embodied in the separation ansatz we would have two separate variation laws which in a fixed background would take the form $c^{2} \delta \rho_{\mathrm{c}}=$ $\Theta \delta s+c^{2} \mu^{\mathrm{c}} \delta n_{\mathrm{c}}$ and $c^{2} \delta \rho_{\mathrm{n}}=c^{2} \mu^{\mathrm{n}} \delta n_{\mathrm{n}}$, in which $\Theta$ would be interpretable as the temperature, $\mu^{\mathrm{c}}$ would be interpretable as the effective mass per baryon in the "normal" part, and $\mu^{\mathrm{n}}$ would be efective mass per neutron in the superfluid part (which would be equal to its analogue in the "normal" part, i.e. $\mu^{\mathrm{n}}=\mu^{\mathrm{c}}$, in the particular case of a state of static thermodynamic equilibrium.)

In the less specialised (liquid type) description to be used here, there will just be a single "conglomerated" variation law, whose most general form, including allowance for a conceivable variation of the background metric, will be expressible (correcting one of the copying errors in the originally published version [56]) as

$$
\delta \Lambda=-\Theta \delta s+\mu^{\mathrm{c}}{ }_{\nu} \delta n_{\mathrm{c}}^{\mu}+\mu^{\mathrm{n}}{ }_{\nu} \delta n_{\mathrm{n}}^{\nu}+\frac{1}{2}\left(c^{-2} \Theta s u_{\mathrm{c}}^{\mu} u_{\mathrm{c}}^{\nu}+n_{\mathrm{c}}{ }^{\mu} \mu^{\mathrm{c} \nu}+n_{\mathrm{n}}{ }^{\mu} \mu^{\mathrm{n} \nu}\right) \delta g_{\mu \nu},
$$

where $\Theta$ is to be interpreted as the temperature and where $\mu_{\mu}^{\mathrm{n}}$ and $\mu_{\mu}^{\mathrm{c}}$ are to be interpreted as the 4-momentum per baryon of the neutron superfluid and the "normal" constituent respectively.

To obtain suitable fluid type dynamical equations from a Lagrangian expressed as above just in terms of the relevant currents, the variation of the latter must be appropriately constrained in the manner 73 that was originally introduced for the case of a simple perfect fluid by Taub 83]. The standard Taub procedure can be characterised as the requirement that the variation of the relevant current three form, which for the "normal" constitituent in the present application will be given in terms of the antisymetric space-time measure tensor $\varepsilon_{\mu \nu \rho \sigma}$ by

$$
N_{\mu \nu \rho}=\varepsilon_{\mu \nu \rho \sigma} n_{\mathrm{c}}^{\sigma},
$$

should be given by Lie transportation with respect to an associated, freely chosen, displacement vector field $\zeta^{\mu}$ say. This ansatz gives the well known result

$$
\delta N_{\mu \nu \rho}=\zeta^{\lambda} \nabla_{\lambda} N_{\mu \nu \rho}+3 N_{\lambda[\mu \nu} \nabla_{\rho]} \zeta^{\lambda} .
$$

Although a variation $\delta g_{\mu \nu}$ of the metric has no effect on the fundamental current three form, $N_{\mu \nu \rho}$, it will contribute to the variation of the corresponding vector,

$$
n_{\mathrm{c}}^{\mu}=\frac{1}{3 !} \varepsilon^{\mu \nu \rho \sigma} N_{\nu \rho \sigma}
$$

for which one obtains

$$
\delta n_{\mathrm{c}}^{\mu}=\zeta^{\nu} \nabla_{\nu} n_{\mathrm{c}}^{\mu}-n_{\mathrm{c}}^{\nu} \nabla_{\nu} \zeta^{\mu}+n_{\mathrm{c}}^{\mu}\left(\nabla_{\nu} \zeta^{\nu}-\frac{1}{2} g^{\nu \rho} \delta g_{\nu \rho}\right)
$$


(Application of an analogous procedure to the entropy current provides the variation rule (96) that was used in Section [3).) In terms of the orthogonally projected metric,

$$
\gamma_{\mathrm{c}}^{\mu \nu}=g^{\mu \nu}+c^{-2} u_{\mathrm{c}}^{\mu} u_{\mathrm{c}}^{\nu},
$$

the corresponding variation of the unit flow vector will be given by

$$
\delta u_{\mathrm{c}}{ }^{\mu}=\gamma_{\mathrm{c}}{ }^{\mu}{ }_{\rho}\left(\zeta^{\nu} \nabla_{\nu} u_{\mathrm{c}}^{\rho}-u_{\mathrm{c}}^{\nu} \nabla_{\nu} \zeta^{\rho}\right)+\frac{1}{2} c^{-2} u_{\mathrm{c}}^{\mu} u_{\mathrm{c}}^{\nu} u_{\mathrm{c}}^{\rho} \delta g_{\nu \rho},
$$

and the corresponding variation in the current amplitude $n_{\mathrm{c}}$ will be

$$
\delta n_{\mathrm{c}}=\nabla_{\nu}\left(n_{\mathrm{c}} \zeta^{\nu}\right)+n_{\mathrm{c}}\left(c^{-2}{u_{\mathrm{c}}}^{\mu}{u_{\mathrm{c}}}^{\nu} \nabla_{\mu} \zeta_{\nu}-\frac{1}{2}{\gamma_{\mathrm{c}}}^{\mu \nu} \delta g_{\mu \nu}\right) .
$$

Since the entropy flux is to be considered as comoving with the "normal" constituent, it is subject to a variation given by the same displacement vector $\zeta$, which thus gives

$$
\delta s=\nabla_{\nu}\left(s \zeta^{\nu}\right)+s\left(c^{-2} u_{\mathrm{c}}{ }^{\mu} u_{\mathrm{c}}{ }^{\nu} \nabla_{\mu} \zeta_{\nu}-\frac{1}{2} \gamma_{\mathrm{c}}^{\mu \nu} \delta g_{\mu \nu}\right) .
$$

On the other hand for the superfluid constituent there will be an independent displacement vector field $\xi^{\mu}$ say, in terms of which the analogously constructed variation will be

$$
\delta n_{\mathrm{n}}^{\mu}=\xi^{\nu} \nabla_{\nu} n_{\mathrm{n}}^{\mu}-n_{\mathrm{n}}^{\nu} \nabla_{\nu} \xi^{\mu}+n_{\mathrm{n}}^{\mu}\left(\nabla_{\nu} \xi^{\nu}-\frac{1}{2} g^{\nu \rho} \delta g_{\nu \rho}\right)
$$

The effect of this variation process on the Lagrangian density $\|g\|^{1 / 2} \Lambda$ itself can be seen to be expressible in the standard form

$$
\|g\|^{-1 / 2} \delta\left(\|g\|^{1 / 2} \Lambda\right)=\zeta^{\nu} f^{\mathrm{c}}{ }_{\nu}+\xi^{\nu} f^{\mathrm{n}}{ }_{\nu}+\frac{1}{2} T^{\mu \nu} \delta g_{\mu \nu}+\nabla_{\mu} \mathcal{R}^{\mu}
$$

in which $f^{\mathrm{c}}{ }_{\nu}$ will be interpretable as the force density acting on the "normal" constituent, $f^{\mathrm{n}}{ }_{\nu}$ will be interpretable as the force density acting on the superfluid constituent, and $T^{\mu \nu}$ will be interpretable as the stress momentum energy density of the two constituent as a whole. By considering the trivial case in which there is no actual physical alteration of the system, but in which the apparent changes are merely due to the displacement of the reference system generated by a vector field $\xi^{\nu}=\zeta^{\nu}$, in which case the apparent variation of the metric will be given by $\delta g_{\nu \nu}=2 \nabla_{[\mu} \zeta_{\nu]}$, it can be seen from (127) that the separate forces must automatically satisfy an identity of the form

$$
f_{\nu}^{\mathrm{c}}+f_{\nu}^{\mathrm{n}}=f_{\nu}^{\mathrm{ex}}
$$

where $f_{\nu}^{\mathrm{ex}}$ is the conglomerated external force density that is defined by

$$
f_{\nu}^{\mathrm{ex}}=\nabla_{\mu} T_{\nu}^{\mu} .
$$

The residual current $\mathcal{R}^{\mu}$ in the divergence will be of no importance for our present purpose (by Green's theorem it just gives a surface contribution that will vanish by the variational boundary conditions) but it is to be noted for the record that it will have the form

$$
\mathcal{R}^{\mu}=2 \zeta^{[\mu} u_{\mathrm{c}}{ }^{\nu]}\left(c^{-2} \Theta s u_{\mathrm{c} \nu}+n_{\mathrm{c}} \mu_{\nu}^{\mathrm{c}}\right)+2 \xi^{[\mu} n_{\mathrm{n}}{ }^{\nu]} \mu^{\mathrm{n}}{ }_{\nu} .
$$

The conglomerated stress momentum energy density tensor can easily be read out as

$$
T^{\mu}{ }_{\nu}=\Psi g^{\mu}{ }_{\nu}+c^{-2} \Theta s u_{\mathrm{c}}{ }^{\mu} u_{\mathrm{c} \nu}+n_{\mathrm{c}}{ }^{\mu} \mu^{\mathrm{c}}{ }_{\nu}+n_{\mathrm{n}}{ }^{\mu} \mu^{\mathrm{n}}{ }_{\nu},
$$


where

$$
\Psi=\Lambda+s \Theta-n_{\mathrm{c}}{ }^{\nu} \mu^{\mathrm{c}}{ }_{\nu}-n_{\mathrm{n}}{ }^{\nu} \mu^{\mathrm{n}}{ }_{\nu} .
$$

(Although this expression is not manifestly symmetric, the asymmetric contributions will automatically cancel due to the identity $\mu^{\mathrm{c}\left[\mu_{n}\right.} n_{\mathrm{c}}{ }^{\nu]}=-\mu^{\mathrm{n}\left[\mu_{n} n_{\mathrm{n}}{ }^{\nu]}\right.}$ ). What matters most for our present purpose is the form of the respective force densities: the force law (i.e. the relevant relativistic generalisation of Newton's "second" law of motion) for the "normal" constituent is found to take the form

$$
f^{\mathrm{c}}{ }_{\nu}=2 s^{\mu} \nabla_{[\mu}\left(c^{-2} \Theta u_{\mathrm{c} \nu]}\right)+2 n_{\mathrm{c}}{ }^{\mu} \nabla_{[\mu} \mu_{\nu]}^{\mathrm{c}}+c^{-2} \Theta u_{\mathrm{c} \nu} \nabla_{\mu} s^{\mu}+\mu_{\nu}^{\mathrm{c}} \nabla_{\mu} n_{\mathrm{c}}^{\mu},
$$

while the force law for the superfluid component is found to take the simpler form

$$
f_{\nu}^{\mathrm{n}}=f_{\nu}^{\mathrm{ch}}+f_{\nu}^{\mathrm{me}}
$$

in which the first term is a "chemical" contribution, representing the effect of any neutron superfluid particle creation or destruction, which is given by

$$
f_{\nu}^{\mathrm{ch}}=\mu_{\nu}^{\mathrm{n}} \nabla_{\mu} n_{\mathrm{n}}^{\mu}
$$

The last term in (134) is a "mechanical" contribution, allowing for drag or pinning forces exerted on the vortices by the crust and balanced by the Magnus effect, according to the formula

$$
f_{\nu}^{\mathrm{me}}=n_{\mathrm{n}}{ }^{\mu} w_{\mu \nu}^{\mathrm{n}}
$$

using the notation

$$
w_{\mu \nu}^{\mathrm{n}}=2 \nabla_{[\mu} \mu_{\nu]}^{\mathrm{n}}
$$

for the vorticity 2 -form of the superfluid neutrons. It is to be noted that this is not the mesoscopic (intervortex) superfluid vorticity, which simply vanishes, but the average vorticity on a macroscopic scale that is large compared with the spacing (typically a very small fraction of a cm.) between the superfluid vortices. For a very accurate treatment it would be necessary to take account of the macroscopic anisotropy resulting from the effective tension of these vortices, as has already been done [54, 55] for the case a single constituent, but for the discussion of global evolution on timescales long compared with the stellar oscillation periods (a small fraction of a second) such an effect seems unlikely to be important.

Although the complete expression (133) is not so simple, it is to be observed that the time component in the "normal" rest frame (representing the rate of working on the "normal" constituent) as obtained by contraction with the relevant unit vector $u_{\mathrm{c}}{ }^{\nu}$ has the comparitively simple form

$$
u_{\mathrm{c}}{ }^{\nu} f^{\mathrm{c}}{ }_{\nu}=u_{\mathrm{c}}{ }^{\nu} \mu^{\mathrm{c}}{ }_{\nu} \nabla_{\mu} n_{\mathrm{c}}{ }^{\mu}-\Theta \nabla_{\mu} s^{\mu} .
$$

If we were to impose the variation principle to the effect that the system should be invariant with respect to arbitrary worldline displacements (as specified by the independent fields $\zeta^{\nu}$ and $\xi^{\nu}$ ) it would follow that each of the forces $f_{\mu}^{\mathrm{c}}$ and $f_{\nu}^{\mathrm{n}}$ would have to vanish. However it is evident from the identity (128) that we cannot adopt such a restrictive postulate in a model designed to treat the effect of pulsar slowdown due to a torque attributable to coupling to an external electromagnetic field that is removing angular momentum by radiation to infinity. As well as the intrinsically non-conservative magnetic torque contribution to $f_{\nu}^{\text {ex }}$ it is also important [40] to include a contribution to allow for the effect of the elastic solidity in the 
crust, which is not incorporated into the simple fluid type model included here (and which would require the use of a much more elaborate model [73] for its detailed evaluation).

Although our ultimate purpose is to allow for a non vanishing external torque force, whatever force law we assume must be such that if the external force $f_{\nu}^{\text {ex }}$ were somehow switched off so as to leave an effectively isolated system, the second law of thermodynics (no decrease of entropy in an isolated system) would be respected, i.e. we must have $f_{\nu}^{\mathrm{ex}}=0 \Rightarrow$ $\nabla_{\nu} d^{\nu} \geq 0$. It can be seen that this is equivalent to the requirement of positivity of the right hand side of the identity

$$
\Theta \nabla_{\mu} s^{\mu}+u_{\mathrm{c}}^{\nu} f_{\nu}^{\mathrm{ex}}=u_{\mathrm{c}}^{\nu}\left(\mu_{\nu}^{\mathrm{n}}-\mu_{\nu}^{\mathrm{c}}\right) \nabla_{\mu} n_{\mathrm{n}}^{\mu}+u_{\mathrm{c}}^{\nu} f_{\nu}^{\mathrm{me}}
$$

that is obtained from (138), taking account of the total baryon conservation law (112). Since they involve very different physical processes, one comes to the conclusion that each of the two terms on the right of (139) must satisfy its own separate positivity condition.

The positivity requirement for the first of these terms is presumably to be attributed to a crust particle creation law of the form

$$
\nabla_{\mu} n_{\mathrm{n}}^{\mu}=\Xi u_{\mathrm{c}}^{\nu}\left(\mu_{\nu}^{\mathrm{n}}-\mu_{\nu}^{\mathrm{c}}\right)
$$

for some positive coefficient $\Xi$. Such a law is an obviously natural generalisation of the kind of creation rate formula that is familiar in chemical physics. In the present context what is involved is conversion of protons to neutrons by weak interactions, and the situation is complicated by the consideration that as far as the large scale mechanics of the neutron star is concerned, the effective rate may depend not just on microscopic processes, but also, when subduction is involved, on the rather messy process whereby the crust is broken up before it ultimately dissolves.

To complete the specification of the system, all that remains is to find the appropriate ansatz for the mechanical force $f_{\nu}^{\mathrm{me}}$. This problem is more delicate than that of the (effectively scalar) chemical case, since as well as the "second law" requirement $u_{\mathrm{c}}^{\nu} f_{\nu}^{\mathrm{me}} \geq 0$, the answer must respect the nature of the macroscopic vorticity 2 -form $w_{\mu \nu}^{\mathrm{n}}$ which although non vanishing (unlike the mesoscopic vorticity between vortices) cannot be arbitrary (as in an ordinary viscous fluid): to be consistent with the underlying superfluid nature of the neutron constituent, it must satisfy an algebraic degeneracy condition of the form (66) in order to be compatible with the existence of a well defined congruence of orthogonal 2-surfaces generated by (non vanishing) tangent vectors, $v^{\nu}$ say, such that $w_{\mu \nu}^{\mathrm{n}} v^{\nu}=0$. It can be seen from the form of the defining relation (136) that the obvious way to obtain this degeneracy property is to take the force law to have the form $f_{\nu}^{\mathrm{me}}=w_{\nu \sigma}^{\mathrm{n}} v^{\sigma}$ for some suitably chosen vector $v^{\nu}$ which, to satisfy the "second law" requirement must satisfy $u_{\mathrm{c}}^{\nu} w_{\nu \sigma}^{\mathrm{n}} v^{\sigma} \geq 0$. The required ansatz can thus be taken to be given by $v_{\mu}=\alpha w_{\mu \nu}^{\mathrm{n}} u_{\mathrm{c}}^{\nu}$ for some positive coefficient $\alpha$. This result is conveniently expressible in terms of the rank-2 tensor $\perp_{\nu}^{\mu}$ of orthogonal projection with respect to the vortex 2 -surface, which is given by

$$
\perp_{\nu}^{\mu}=2\left(w^{\mathrm{n} \rho \sigma} w_{\rho \sigma}^{\mathrm{n}}\right)^{-1} w^{\mathrm{n} \lambda \mu} w_{\lambda \nu}^{\mathrm{n}} .
$$

We end up with an expression taking the form

$$
f_{\nu}^{\mathrm{me}}=\eta_{\mathrm{r}} \perp_{\nu \sigma} u_{\mathrm{c}}^{\sigma}
$$


for a positive resistive drag coefficient $\eta_{\mathrm{r}}$ (given in terms of the previous coefficient $\alpha$ by $2 \eta_{\mathrm{r}}$ $\left.=\alpha w^{\mathrm{n} \rho \sigma} w_{\rho \sigma}^{\mathrm{n}}\right)$.

The generic class of dissipative models characterised by finite values of $\Xi$ and $\eta_{\mathrm{r}}$ has four different kinds of non dissipative limit. In the low reactivity limit $\Xi \rightarrow 0$ we have the non-transfusive limit characterised by the separate superfluid particle conservation law

$$
\nabla_{\nu} n_{\mathrm{n}}^{\nu}=0
$$

whereas in the opposite high reactivity limit $\Xi \rightarrow \infty$ we have the chemical equilibrium limit characterised by

$$
u_{\mathrm{c}}^{\nu}\left(\mu_{\nu}^{\mathrm{c}}-\mu_{\nu}^{\mathrm{n}}\right)=0
$$

which is what would be expected in cases for which the (continental drift like) crust circulation responsible for the transfusion is characterised by timecales that are very long compared with those 98 of the relevant weak (direct or inverse beta decay) interactions. For each of these conceivably relevant possibilities, we have the drag free limit $\eta_{\mathrm{r}} \rightarrow 0$ characterised by the condition of vanishing Magnus force,

$$
n_{\mathrm{n}}^{\mu} w_{\mu \nu}^{\mathrm{n}}=0
$$

or at the opposite extreme the perfect vortex pinning limit, $\eta_{\mathrm{r}} \rightarrow \infty$ characterised by the condition that the vortex worldsheets should be at rest with respect to the "normal" (crust) background,

$$
u_{\mathrm{c}}^{\mu} w_{\mu \nu}^{\mathrm{n}}=0 .
$$

The actual evaluation of the drag coefficient $\eta_{\mathrm{r}}$, and the question of whether one or other of these simple extreme limits is realistic depends on delicate technical issues [96, 97] whose definitive resolution is not entirely clear. A scenario of the type envisaged by Ruderman [39], as represented by Figure 1, and described by (39) (which seems to be appropriate for Vela) is what would be obtained in the case characterised by (146), whereas the more recently proposed alternative scenario [40] represented by Figure 2, and described by (40), is what would be obtained in the case characterised by (145).

It is of course to be expected that such extreme scenarios will turn out in practise to be oversimplifications of a more complicated reality, whose description is likely to require modelisation with not just two 61] but many independently rotating components, to allow for the variation of the chemical and mechanical coefficients $\Xi$ and $\eta_{\mathrm{r}}$ over a wide range of finite values as a function of depth in the star.

Acknowledgements The author wishes to thank Silvano Bonazzola, David Langlois, Eric Gourghoulon, Pawel Haensel, Isaac Khalatnikov, Reinhardt Prix, and David Sedrakian for conversations and collaboration. 


\section{References}

[1] B.K. Harrison, K.S. Thorne, M. Wakano, J.A. Wheeler, Gravitation theory and gravitational collapse (University of Chicago Press, Chicago, 1965).

[2] A.B. Migdal, "Superfluidity of the moments of inertia of nuclei", Nucl. Phys. 13, 655-674 (1959).

[3] N. K. Glendenning, Compact stars (Springer, Heidelberg, 1997).

[4] F. Weber, "Pulsars as astrophysical laboratories for nuclear and particle physica" (I.O.P. Publishing, Bristol, 1999).

[5] D. Langlois, "Superfluidity in relativistic neutron stars". astro-ph/0008161]

[6] L.D. Landau, E.M. Lifshitz, Course of Theorteical Physics, Vols. 1 - 9 (Pergamon, Oxford, 1959).

[7] G. Baym, D. Pines, "Neutron starquakes and pulsar speedup", Annals of Physics 66, 816-835 (1971).

[8] G. Baym, C. Pethick, D. Pines, M. Ruderman, "Spin up in neutron stars: the future of the Vela pulsar", Nature 224, 872-874 (1969).

[9] M. Hoffberg, A.E. Glassgold, R.W. Richardson, M. Ruderman, "Anisotropic superfluidity in neutron star matter", Phys. Rev. Lett. 24, 775-777 (1970).

[10] J.A. Sauls, D.L. Stein, J.W. Serene, "Magnetic vortices in a ${ }^{3} P_{2}$ neutron superfluid", Phys. Rev. D25, 967-975 (1982).

[11] N.K. Glendenning, "First order phase transitions with more than one conserved charge: consequences for neutron stars", Phys. Rev. D46, 1274-1287 (1992).

[12] N.K. Glendenning, S. Pei, "Crystalline structure of the mixed confined - deconfined phase in neutron stars", Phys. Rev. C52, 2250-2253 (1995).

[13] M. Alford, K. Rajagopal, F. Wilczek, "Color-flavor locking and chiral symmetry breaking in high density QCD", Nucl. Phys. B537, 443-458 (1999). hep-th/9804403

[14] D. Blaschke, D.M. Sedrakian, K.M. Shahabasyan, "Diquark condensates and the magnetic field of pulsars", Astron. Astrophys. 350, L47 (1999). astro-ph/9904395

[15] M. Alford, J. Berges, K. Ragjagopal, "Magnetic fields within colour superconducting neutron star cors". hep-ph/9910254

[16] D.M. Sedrakian, D. Blaschke, K.M. Shahabasyan, D.N. Voskresensky, "Meissner effect for color superconducting quark matter". hep-ph/0012383]

[17] B. Carter, "Application of continuous variation formulas and discrete invariance principles to black holes and neutron star models", Annals of Physics, 95, 53 -73 (1974).

[18] G.A. Vardian, D.M. Sedrakian, "Magnetohydrodynamics of superfluid solutions", Sov. Phys. J.E.T.P. 54(5), 919-921 (1981). 
[19] M.A. Alpar, S.A. Langer, J.A. Sauls, "Rapid postglitch spin - up of the superfluid core in pulsars", Astroph. J. 282, 533-541 (1984).

[20] S.E. Thorsett, D.Chakrabarty, "Neutron star mass measurements: radio pulsars", Astroph. J. 512, 288-299 (1999).

[21] E. Chubarian, H. Grigorian, G. Poghosyan, D. Blaschke, "Deconfinement transition in rotating compact stars", Astron. Astrophys. 357, 968-976 (2000). astro-ph/9903489

[22] M. Ruderman, "Neutron starquakes and pulsar periods", Nature 223, 597-598 (1969).

[23] R. Smoluchowski, "Frequency of pulsar starquakes", Phys. Rev. Lett. 24, 923-925 (1970).

[24] P.W. Anderson, N. Itoh, "Pulsar glitches and restlessness as a hard superfluidity phenomenon", Nature 256, 25-26 (1975).

[25] A. Sedrakian, J.M. Cordes, "Spin evolution of pulsars with weakly coupled superfluid interiors", Astroph. J. 502, 378-381 (1989). astro-ph/9802102

[26] M.A. Alpar, P.W. Anderson, D. Pines, J. Shaham, "Giant glitches and pinned vorticity in the Vela and other pulsars", Astroph. J. Lett. 249, L29 (1981).

[27] A. Sedrakian, I. Wasserman, J.M. Cordes, "Precession of isolated neutron stars. 1. Effects of imperfect pinning", Astroph. J. 524, 341-360 (1999).

[28] M.Q. Alpar, J.A. Sauls, "On the dynamical coupling between the superfluid interior and the crust of a neutron star" Astroph. J. 327, 723-725 (1988).

[29] L. Bildsten, R.I. Epstein, "Superfluid dissipation timescales in neutron star crusts", Astroph. J., 342, 951-957 (1989)

[30] A.D. Sedrakian, D.M. Sedrakian, "Non - stationary dynamics of rotating superfluid systems", Sov. Phys. JETP 75, 395-399 (1992).

[31] M.A. Alpar, P.W. Anderson, D. Pines and J. Shaham, "Vortex creep and the internal temperature of neutron stars", Astroph. J. 276, 325-334 (1984); Astroph. J. 278, 791-805 (1984).

[32] P.B. Jones, "Rotation of the neutron drip superfluid in pulsars: period discontinuities and internal temperatures", Mon. Not. R. Astr. Soc. 246, 315-323 (1990).

[33] A.D. Sedrakian and D.M. Sedrakian, "Superfluid core rotation in pulsars", Astroph. J. 447, 305-323 (1995).

[34] P.B. Jones, "Rotation of the neutron drip superfluid in pulsars: the interaction and pinning of vortices" Astroph. J. 372, 208-212 (1991).

[35] B.K. Link, R.I. Epstein, "Mechanics and energetics of vortex pinning in neutron stars", Astroph. J. 373, 592-603 (1991).

[36] B.K. Link, R.I. Epstein, "Thermally driven neutron star glitches", Astroph. J. 457, 844854 (1996). 
[37] A. Sedrakian, J.M. Cordes, "Vortex - interface interactions and generation of glitches", Mon. Not. R. Astr. Soc. 307, 365 (1999). astro-ph/9806042]

[38] M. Ruderman, "Crust breaking by neutron superfluids and the Vela pulsar glitches", Astroph. J. 203, 213-222 (1976).

[39] M. Ruderman, "Neutron star crustal plate tectonics. I. Magnetic dipole evolution in millisecond pulsars and low mass X-ray binaries", Astroph. J. 366, 261-269 (1991).

[40] B. Carter, D. Langlois, D.M. Sedrakian, "Centrifugal buoyancy as a mechanism for neutron star glitches", Astron. Astroph 361, 795-802 (2000). astro-ph/0004121.

[41] R. Prix, "Slowly rotating two-fluid neutron star models", Astron. Astrophys. 352, 623631 (1999). [astro-ph/9910293].

[42] S.L. Shapiro, S.A. Teukolsky, Black holes, white dwarfs, and neutron stars (Wiley, New York, 1883).

[43] F. Pacini, "Energy emission from a neutron star", Nature, 216, 567-568 (1967).

[44] P. Goldreich, W.H. Julian, "Pulsar electrodynamics", Astroph. J. 157, 869 (1969).

[45] N. K. Glendenning, S. Pei, F. Weber, "Signal of quark deconfinement in the timing structure of pulsar spin down", Phys. Rev. Lett. 79 , 1603-1606 (1997).

[46] V.S. Beskin, A.V. Gurevich, Ya. N. Istomin, Physics of the pulsar magnetosphere (C.U.P., Cambridge, 1993).

[47] A. Melatos, "Spin down of an oblique rotator with a current starved outer magnetosphere", Mon. Not. R. Astr. Soc. 288, 1049-1059 (1997).

[48] A.G. Lyne, R.S. Pritchard, F.G. Smith, "Crab pulsar timing 1982 - 87", Mon. Not. R. Astr. Soc. 233, 667-676 (1988).

[49] A.G. Lyne, R.S. Pritchard, F.G. Smith, F. Camilio, "Very low braking index for the Vela pulsar", Nature 381, 497-498 (1996).

[50] J. Shaham, "Free precession of neutron stars: the role of possible vortex pining", Astroph. J. 214, 251-260 (1977).

[51] A. Melatos, "Radiative precession of an isolated neuton star". astro-ph/0004035

[52] B. Carter, H. Quintana, "Relativistic formulation of the neutron starquake theory pulsat glitches", Annals of physica 95, 74-89 (1975).

[53] B. Carter, H. Quintana, "Stationary elastic deformation of a relativistic neutron star model", Astroph. J. 202, 511-522 (1975).

[54] B. Carter, D. Langlois, "Kalb-Ramond coupled vortex fibration model for relativistic superfluid dynamics", Nuclear Physics B454, 402-424 (1995). hep-th/9611082 
[55] B. Carter, "Relativistic dynamics of vortex defects in superfluids" in Topological defects and the non-equilibrium dynamics of symmetry breaking phase transitions (Les Houches 99), ed. Y.M. Bunkov and H. Godfrin, pp 267-301 (Kluwer, 2000). gr-qc/9907039

[56] D. Langlois, D. M. Sedrakian, B. Carter, "Differential rotation of relativistic superfluid in neutron stars" Mon. Not. Roy. Astr. Soc. 297, 1198-1201 (1998). astro-ph/9711042

[57] L. Lindblom, G. Mendel, "The oscillations of superfluid neutron stars", Astroph. J. 421, 689-704 (1994).

[58] U. Lee, "Nonradial oscillations of neutron stars with superfluid cores", Astron. Astrop. 303, 515-525 [1995).

[59] A. Sedrakian, I. Wasserman, "Perturbations of self - gravitating, ellipsoidal superfluid normal fluid mixture", Phys. Rev. D63, 024016 (2000). astro-ph/0004331

[60] G.L. Comer, D. Langlois, L.M. Lin, "Quasi-normal modes of general relativistic neutron stars", Phys. Rev. D60, 104025 (1999). gr-qc/9908040

[61] N. Andersson, G.L. Comer, "Slowly rotating general relativistic superfluid neutron stars". gr-qc/0009089

[62] N. Stergioulas, J. Friedman, "Non axisymmetric neutral modes in rotating relativistic stars", Astroph. J. 492, 301-322 (1998). gr-qc/9705056

[63] S. Bonazzola, J. Frieben, E. Gourgoulhon, "Spontaneous symmetry breaking of rapidly rotating stars in general relativity", Astron. Astrophys. 331, 280-290 (1998). grqc/9710121]

[64] S. Morsink, N. Stergioulas, S.R. Blattnig, "Quasi - normal modes of rotating relativistic stars - neutral modes for realistic equations of state", Astroph. J. 510, 854-861 (1999). gr-qc/9806008

[65] E. Gourgoulhon, P. Haensel, R. Levine, E. Paluch, S. Bonazzola, J.A. Marck, "Fast rotation of strange stars", Astron. Astroph. 349, 851-862 (1999). astro-ph/9907225

[66] N. Andersson, D.I. Jones, K.D. Kokkotas, N. Stergioulas, "R-mode runaway and rapidly rotating neutron stars", Astroph. J. 534, L75 (2000). astro-ph/0002114

[67] G.E. Volovik, "Exotic properties of superfluid helium 3" (World Scientific, Singapore, 1992).

[68] J.A. Sauls "Superfluidity in the interior of neutron stars" in Timing Neutron Stars, Nato ASI C262, ed. H. Ogelman, E.J.P. van den Heuvel. pp 457-490 (Kluwer, Dordrecht, 1988).

[69] G. Mendel, L. Lindblom, "The coupling of charged superfluid mixtures to the electromgnetic field", Annals of Physics 205, 110-129 (1991).

[70] B. Carter, D. Langlois, "Relativistic models for superconducting superfluid mixtures", Nuclear Phys. B531, 478-504 (1998). gr-qc/9806024 
[71] B. Carter, "Superconducting superfluids in neutron stars", Gravitation and Cosmology 6, Supplement, 204-213 (2000). astro-ph/0010109

[72] I.L. Bekarevich, I.M. Khalatnikov, "Phenomenological derivation of vortex motion in HeII", Sov. Phys. J.E.T.P. 13, 643-646 (1961).

[73] B. Carter, "Covariant Theory of Conductivity in Ideal Fluid or Solid Media", in Relativistic Fluid Dynamics (C.I.M.E., Noto, May 1987) ed. A.M. Anile, \& Y. Choquet-Bruhat, Lecture Notes in Mathematics 1385 pp 1-64 (Springer - Verlag, Heidelberg, 1989).

[74] V.V. Lebedev, I.M. Khalatnikov, "Relativistic hydrodynamics of superfluids", Sov. Phys. J.E.T.P. 56, 923-930 (1982).

[75] B. Carter, I.M. Khalatnikov, "Equivalence of convective and potential derivations of covariant superfluid dynamics", Phys. Rev. D45, 4536-4544 (1992).

[76] B. Carter, D. Langlois, "The Equation of state for cool relativistic two-constituent superfluid dynamics", Phys. Rev. D51, 5855-5864 (1995). [hep-th/9507 058]

[77] B. Carter, "Perfect fluid and magnetic field conservation laws in the theory of black hole accretion rings", B. Carter in Active Galactic Nuclei, ed. C. Hazard \& S. Mitton, pp 273-300 (Cambridge U.P., 1979).

[78] B. Carter, "Black hole equilibrium states", in Black Holes (proc. 1972 Les Houches Summer School), ed. B. \& C. DeWitt, pp 57-210 (Gordon and Breach, New York, 1973).

[79] W. Unruh, "Experimental black hole evapouration", Phys. Rev. Letters 46, 1351-1357 (1981).

[80] W. Unruh, "Dumb holes and the effects of high frequencies on black hole evapouration", Phys. Rev. D51, 2827-2838 (1995). gr-qc/9409008

[81] C. Barcelo, S. Liberati, M. Visser, "Analog gravity from Bose-Einstein condensates". gr-qc/0011026

[82] A. Lichnerowicz. Relativistic Hydrodynamics and Magnetohydrodynamics (Benjamin, New York, 1967).

[83] A.H. Taub, "General relativistic variation principle for perfect fluids", Phys. Rev. 94, 1469-1470 (1954).

[84] B. Schutz, "Perfect fluids in general relativty: velocity potentials and a variational principle", Phys. Rev. D2, 2762-2773 (1970).

[85] B. Carter, "Axionic Vorticity Variational formulation for Relativistic Perfect Fluids", Class. Quantum Grav. 11, 2013-2030 (1994).

[86] B.Carter, in A Random Walk in Relartivity and Cosmology, Proc. Vadya - Raychaudhuri Festschrift, IAGRG 1983, ed. N. Dadhich, J. Krishna Rao, J.V. Narlikar, C.V. Vishveshwara, pp 48 -62 (Wiley Eastern, Bombay, 1985). 
[87] B. Carter, I.M. Khalatnikov, "Momentum, Vorticity, and Helicity in Covariant Superfluid Dynamics", Ann. Phys. 219, 243-265 (1992).

[88] W. Israel, "Covariant superfluid dnamics", Physics Letters A86, 79-81 (1981); "Equivalence of two theories of relativistic superfluid dynamics", Physics Letters A92, 77-78 (1982)

[89] W.G. Dixon, Arch. Rat. Mech. Anal. 80, 159 (1982).

[90] T.S. Olsen, "Instabilities in the Israel-Dixon relativistic superfluid theory", Physics Letters A149, 71-75 (1990).

[91] L.D. Landau, E.M. Lifshitz, Statistical Physics (trad. E. and R.F. Peierls), Section VI (Pergamon, Oxford, 1959).

[92] R.I. Epstein, "Acoustic properties of neutron stars" Astroph. J. 333, 880-894 (1988).

[93] S. Tsuruta, "Thermal properties and detectability of neutron stars", Phys. Rep. 56, 237-278 (1979).

[94] A.F. Andreev, E.P. Bashkin, "Three veloity hydrodynamics of superfluid solutions", Sov. Phys. J.E.T.P. 42, 164-167 (1976).

[95] O. Sjoberg, "On the Landau effective mass in asymmetric nuclear matter", Nucl. Phys. A265, 511-516 (1976).

[96] P. B. Jones, "Rotation of the neutron drip superfluid in pulsars: the Kelvin phonon contribution to dissipation", Mon. Not. R. Astr. Soc., 257, 501-506 (1992).

[97] B. Link, R.I. Epstein, G. Baym, "Superfluid vortex creep and rotational dynamics of neutron stars", Astroph. J., 403, 285-302, (1993).

[98] P. Haensel, "Non-equilibrium neutrino emissivities and opacities of neutron star matter", Astron. Astroph., 262, 131-137 (1992). 